\title{
Role of Mesoscale Eddies in Modulating the Semidiurnal Internal Tide: Observation Results in the Northern South China Sea ${ }^{\mathscr{O}}$
}

\author{
Xiaodong Huang, Zhaoyun Wang, Zhiwei Zhang, Yunchao Yang, Chun Zhou, \\ QINGXUAN YANG, WEI ZHAO, AND JIWEI TIAN \\ Physical Oceanography Laboratory, and Collaborative Innovation Center of Marine Science and Technology, \\ Ocean University of China, and Qingdao National Laboratory for Marine Science and Technology, Qingdao, China
}

(Manuscript received 5 October 2017, in final form 4 May 2018)

\begin{abstract}
The role of mesoscale eddies in modulating the semidiurnal internal tide (SIT) in the northern South China Sea (SCS) is examined using the data from a cross-shaped mooring array. From November 2013 to January 2014, an anticyclonic eddy (AE) and cyclonic eddy (CE) pair crossed the westward SIT beam originating in Luzon Strait. Observations showed that, because of the current and stratification modulations by the eddy pair, the propagation speed of the mode-1 SIT sped up (slowed down) by up to $0.7 \mathrm{~m} \mathrm{~s}^{-1}\left(0.4 \mathrm{~m} \mathrm{~s}^{-1}\right)$ within the AE's (CE's) southern portion. As a result of the spatially varying phase speed, the mode- 1 SIT wave crest was clockwise rotated (counterclockwise rotated) within the AE (CE) core, while it exhibited convex and concave (concave and convex) patterns on the southern and northern peripheries of the AE (CE), respectively. In midto-late November, most of the mode-1 SIT energy was refracted by the AE away from Dongsha Island toward the north part of the northern SCS, which resulted in enhanced internal solitary waves (ISWs) there. Corresponding to the energy refraction, responses of the depth-integrated mode-1 SIT energy to the eddies were generally in phase at the along-beam-direction moorings but out of phase in the south and north parts of the northern SCS at the cross-beam-direction moorings. From late December to early January, intensified mode2 SIT was observed, whose energy was likely transferred from the mode-1 SIT through eddy-wave interactions. The observation results reported here are helpful to improve the capability to predict internal tides and ISWs in the northern SCS.
\end{abstract}

\section{Introduction}

Internal tides (ITs), which are generated by barotropic tides flowing over varying topography, are ubiquitous in global oceans. They transport significant amounts of energy over hundreds to thousands of kilometers away from the generation sites (Alford 2003; Tian et al. 2003; Zhao et al. 2016) and behave as the primary driver of diapycnal mixing in the deep ocean (Egbert and Ray 2000; Munk and Wunsch 1998; Wunsch and Ferrari 2004). Luzon Strait (LS), as the only deep channel connecting the South China Sea (SCS) and the western Pacific, is one of the most energetic internal tide generation sites among global oceans (Zhao 2014).

Supplemental information related to this paper is available at the Journals Online website: https://doi.org/10.1175/JPO-D-170209.s1.

Corresponding author: Wei Zhao, weizhao@ouc.edu.cn
The ITs originating in LS transport about $7 \mathrm{GW}$ energy into the northern SCS (Alford et al. 2011; Jan et al. 2008), which can cause isopycnal displacements as high as $200 \mathrm{~m}$ in this marginal sea (Klymak et al. 2011). Dissipation of these energetic ITs fuels the enhanced diapycnal mixing in the deep SCS (Tian et al. 2009; Yang et al. 2016), which drives a unique cyclonic deep-water circulation in the SCS deep basin (Zhao et al. 2014). In addition, ITs play a decisive role in the formation of high-frequency internal solitary waves (ISWs) in the northern SCS (Buijsman et al. 2010; Farmer et al. 2009; Zhao 2014; Zhao et al. 2004), which are effective in modulating the ocean ecosystem (Wang et al. 2007), transporting energy (Huang et al. 2016; Klymak et al. 2006), and driving mixing in the shallow water (St. Laurent et al. 2011; Lien et al. 2014).

Besides ITs, mesoscale eddies are prevalent in the northern SCS (Wang et al. 2003; Wu and Chiang 2007; Zhang et al. 2017). Statistical analysis of satellite altimeter data from Chen et al. (2011) revealed that 
mesoscale eddies were present during $35 \%-60 \%$ of the time in the northern SCS, suggesting that the encounters of ITs with mesoscale eddies are frequent. In addition, mesoscale eddies are dynamically important in modulating the background current and stratification fields in the northern SCS. Zhang et al. (2013) demonstrated that the low-frequency current velocities in the northern SCS associated with mesoscale eddies were up to $1 \mathrm{~m} \mathrm{~s}^{-1}$ near the surface and could reach $0.1 \mathrm{~m} \mathrm{~s}^{-1}$ at $1000-\mathrm{m}$ depth, and the temperature anomalies associated with anticyclonic eddies (AE) and cyclonic eddies (CE) could be as large as $7.5^{\circ}$ and $-3.0^{\circ} \mathrm{C}$, respectively, near the main thermocline. Given that energetic mesoscale eddies frequently occupy the propagation path of ITs in the northern SCS, a better understanding of how mesoscale eddies modulate the propagation of ITs is fundamental to understand the variations of ITs in this region and is thus helpful to improve their predictions.

Previous observational studies have suggested the potential impacts of mesoscale eddies on the propagation of ITs. Rainville and Pinkel (2006) found that the phase of ITs became random as they propagated away from the Hawaiian Ridge owing to the impacts of mesoscale eddies. Through comparing the semidiurnal IT (SIT) measured from moorings and satellite altimeter, Zhao et al. (2010) reported that phase differences between the two measurements grew with increasing distance from the Hawaiian Ridge, which was possibly due to refraction by mesoscale eddies. Park and Watts (2006) demonstrated that because of the changes in the mesoscale circulation and stratification, the propagation pattern of the SIT varied from month to month in the Japan Sea. Nash et al. (2012) reported that the ITs over the New Jersey shelf were incoherent and largely unpredictable, possibly owing to eddies in their path. The impacts of mesoscale eddies on the propagation of ITs have also been investigated by numerical simulations. Kerry et al. (2014) demonstrated that the spatial patterns of internal tide propagation near LS were influenced by the location of eddies. Dunphy and Lamb (2014) showed that the internal tidal energy was produced in beam-like patterns after passing through mesoscale eddies. Ponte and Klein (2015) demonstrated that internal tide became more incoherent when the background eddy turbulence was strengthened.

As ITs propagate through mesoscale eddies, energy exchange between them may also happen. Chavanne et al. (2010) demonstrated that an eddy caused the amplification of IT energy near the surface by a factor of 15 and the decrease of vertical wavelength by a factor of 6 . Using a mathematical model, Lelong and Riley (1991) demonstrated that wave-wave-vortex triad interactions could be found between two equal-frequency waves and one vortical mode, and the vortex facilitated energy transfer between two wave modes by acting as a catalyst. It was confirmed recently by the numerical simulations performed by Dunphy and Lamb (2014), which showed that a mode- 1 baroclinic eddy could result in the scattering of energy from the incident mode-1 ITs to modes two and higher. However, the formation of higher-mode ITs through the wave-wave-vortex triad interactions still needs to be confirmed by observations.

Although previous studies have suggested the potential impacts of mesoscale eddies on the ITs, to our best knowledge, details of these impacts have never been examined based on in situ observations in the northern SCS. Deeply investigating this issue is important for us to better understand the propagation and fate of ITs originating in LS. In this study, the role of mesoscale eddies in modulating the SIT in the northern SCS is quantitatively investigated using the observations from a cross-shaped mooring array consisting of 11 moorings (Fig. 1), which is located on the westward SIT beam originating in LS (Fig. 7 in Zhao 2014). In addition to ITs and eddies, ISWs, which are developed from the SIT (Buijsman et al. 2010; Farmer et al. 2009; Zhao 2014), are also widely distributed in the northern SCS (Alford et al. 2015; Huang et al. 2014; Ramp et al. 2010; Zhao et al. 2004). In a previous work, we have demonstrated that mesoscale eddies could significantly affect the propagation of ISWs (Huang et al. 2017). Here, we also investigate the variations of ISW formations in the northern SCS due to the interactions between the SIT and mesoscale eddies.

This paper is organized as follows. We introduce the data, oceanographic background, and methods in section 2 . We show the variations in the mode- 1 SIT and ISWs associated with the eddies in section 3 . We discuss the variations of the higher-mode SIT during the eddy period in section 4. A summary is given in section 5 . The deviation of the Taylor-Goldstein equation in the presence of background shear and rotation, as well as how to solve this equation, is described in appendix A. The errors in the energy and flux calculations are presented in appendix B.

\section{Data, oceanographic background, and methods}

\section{a. In situ mooring data}

To investigate the 3D structure and life cycle of mesoscale eddies and their interactions with internal waves, the South China Sea Mesoscale Eddy Experiment (S-MEE) was designed and successfully conducted in the northern SCS during 2013-14 (Huang et al. 2017; Zhang et al. 2016, 2017). During the S-MEE, an array of 


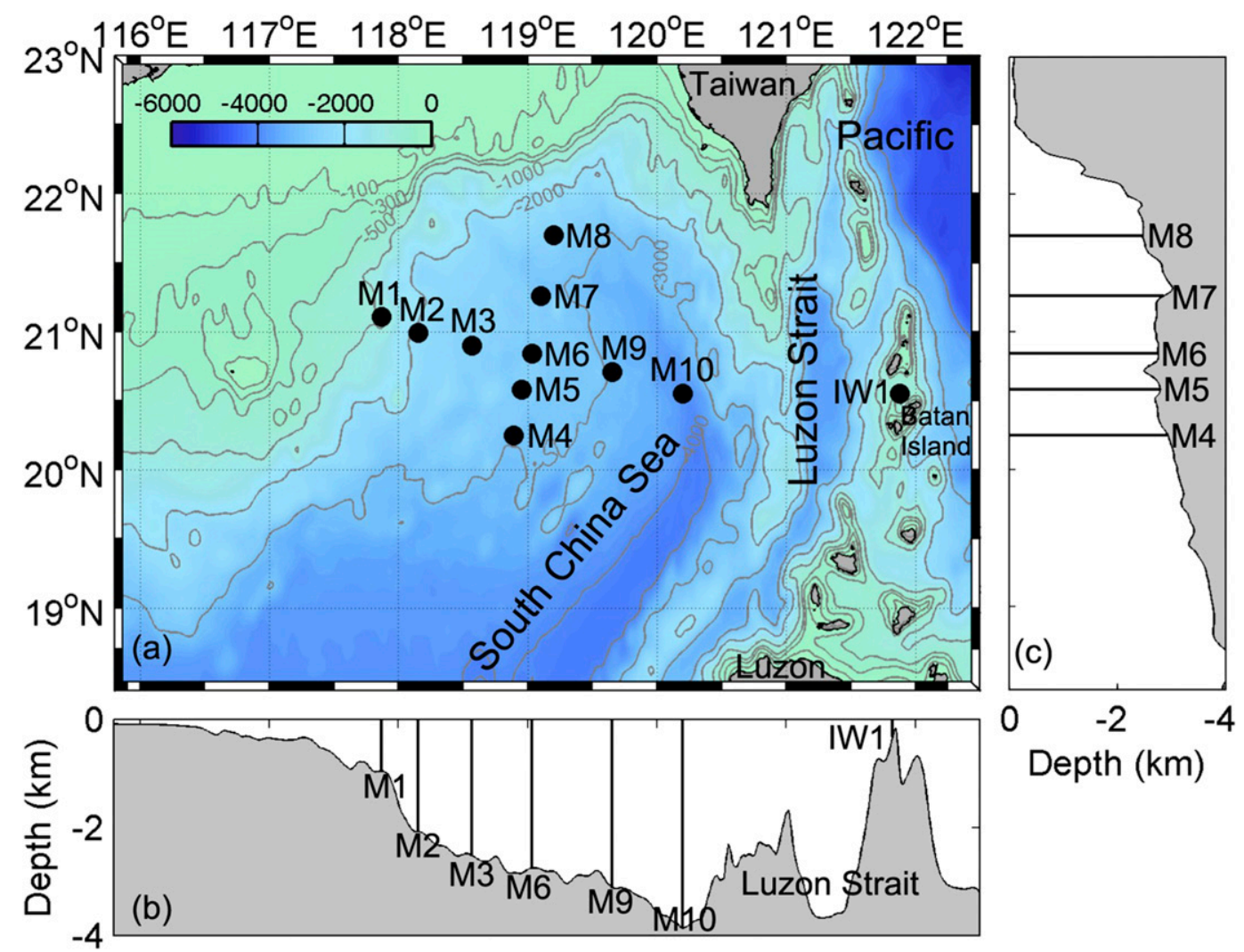

FIG. 1. (a) Topography of the northern South China Sea and Luzon Strait, where the circles denote mooring locations. (b) Bathymetry along the line of mooring in the along-beam direction. (c) Bathymetry along the line of mooring in the cross-beam direction.

11 moorings (Fig. 1) with a cross shape were deployed from late October 2013 to early June 2014. In this mooring array, a line of seven moorings (IW1, M1-M3, M6, M9, and M10; Fig. 1b) were deployed along the westward SIT beam originating in LS as shown in Fig. 7 in Zhao (2014), and the other four moorings (M4, M5, M7, and M8; Fig. 1c) were deployed in the cross-beam direction on the sides of mooring M6.

On each mooring except IW1, two $75-\mathrm{kHz}$ acoustic Doppler current profilers (ADCPs) were mounted at a nominal depth of $\sim 500 \mathrm{~m}$, with one looking upward and the other downward. At IW1 in LS, one upward-looking $75-\mathrm{kHz}$ ADCP was mounted on the mooring at a nominal depth of $279 \mathrm{~m}$. At M2, an additional downwardlooking 75- and 300-kHz ADCP was mounted on the mooring at a nominal depth of 950 and $1920 \mathrm{~m}$, respectively. The ADCPs on the moorings generally had a sampling interval and vertical bin size of $3 \mathrm{~min}$ and $16 \mathrm{~m}$ ( $4 \mathrm{~m}$ for the $300-\mathrm{kHz}$ ADCP), respectively. In addition to the ADCPs, all the moorings except IW1 and M6 were equipped with temperature/salinity $(T / S)$ chains to monitor upper-layer $T / S$. The sampling intervals of $T / S$ chains were set to 2 or $3 \mathrm{~min}$. Moorings M2-M10 were also equipped with several Aanderaa Seaguard recording current meters (RCMs) and CTDs below $\sim 1500 \mathrm{~m}$ to monitor current velocity and $T / S$ in the deep ocean. The instruments mounted on the moorings generally functioned well, except at M5 where the ADCP data were unable to resolve IT signals for unknown reasons. Detailed information of the moorings, including their locations, instrument settings, and working times, are summarized in Table S1 in the supplemental information.

A 5-day segment of the raw data at M2 is shown in Fig. 2. Both upper and lower layers are covered by the current velocity and temperature measurements, although gaps exist near the top and bottom and between the instruments. A rich field of tidal currents can be seen, even at $2000 \mathrm{~m}$, near the bottom. In the temperature measurements, IT motions are also apparent. Besides tides, high-frequency signals associated with ISWs are observed.

\section{b. Mesoscale eddies}

To identify mesoscale eddies, the simultaneous altimeter sea level anomaly (SLA) data and surface 


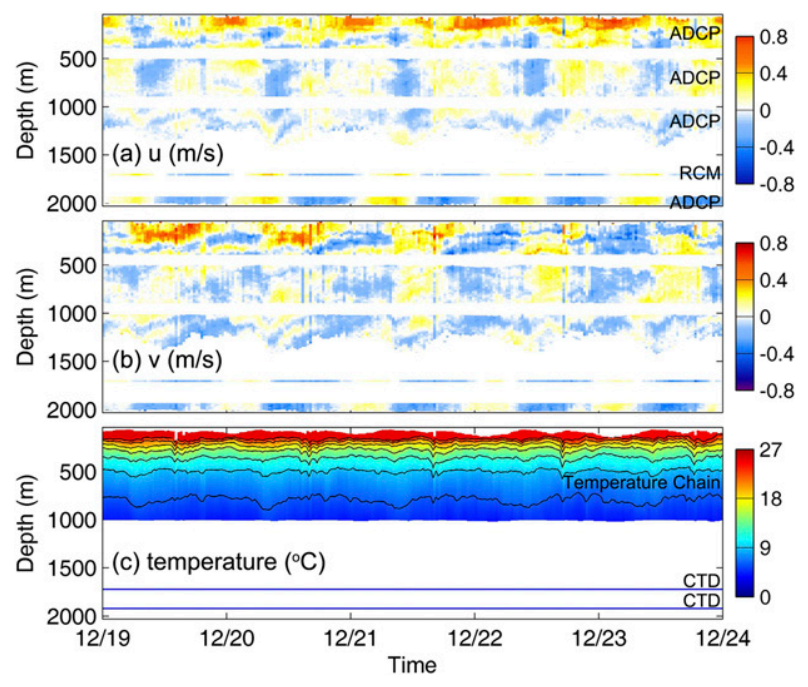

FIG. 2. A 5-day segment of measurements at M2. (a) Zonal velocity. (b) Meridional velocity. (c) Temperature. The black contours represent the isotherms between $6^{\circ}$ and $24^{\circ} \mathrm{C}$ at an interval of $3^{\circ} \mathrm{C}$.

geostrophic velocity field from the Archiving, Validation, and Interpretation of Satellite Oceanographic Data (AVISO) are used. Figure 3 shows that a mesoscale eddy pair consisting of one $\mathrm{AE}$ and one $\mathrm{CE}$ was generated in the region southwest of Taiwan in November/December 2013. After the generation, they propagated southwestward and exactly crossed the mooring array from November 2013 to January 2014.

Figure 4 presents the low-frequency variations (with periods $>2.5$ days) in the current and stratification measurements at M2. It can be seen that the eddy pair caused remarkable changes in both zonal and meridional current components with fluctuation magnitudes of 0.5 and $0.7 \mathrm{~m} \mathrm{~s}^{-1}$, respectively. The current velocity associated with the eddy pair extended from the surface to near bottom, deeper than $2000 \mathrm{~m}$. When the AE passed, the thermocline was depressed remarkably from 11 November to 10 December and then gradually returned to its original depth around 3 January. The influence of the $\mathrm{CE}$ on stratification at M2 was weaker than the AE. The 3D structure, generation, and dissipation of this eddy pair reported here are described in detail in Zhang et al. (2016).

\section{c. Tides in $L S$}

In LS, the primary source of ITs in the northern SCS, the tidal information is obtained from the ADCP measurements near the Batan Island at IW1. At this site, the time series of barotropic current $\overline{\mathbf{u}}(t)$ is estimated by averaging the velocity data between 50 and $250 \mathrm{~m}$, which covers $61 \%$ of water column. The barotropic tidal currents, as well as their diurnal and semidiurnal components, are extracted by harmonic analysis using the T_TIDE toolbox (Pawlowicz et al. 2002). The estimated tidal constants (see Table S2 in the supplemental information) at IW1 agree well with the predictions from the barotropic data-assimilated global tidal model TPXO 7.2 (Egbert and Erofeeva 2002), suggesting that the estimated barotropic tidal current is not significantly contaminated by baroclinic signals due to the incomplete depth coverage of the velocity measurements.

At IW1, the tidal current ellipse major axes are generally in the west-east direction. As shown in Fig. 5a, the zonal barotropic tidal current at IW1 exhibits evident spring-neap tidal cycles with a period of about 14 days. The upper and lower envelopes of both the observed and predicted zonal barotropic tidal current are asymmetrical, showing the ebbing (eastward) dominance of the tidal current. Diurnal and semidiurnal barotropic tidal currents (Figs. 5b,c) also experience spring-neap modulations. When the eddy pair crossed the northern SCS between November 2013 and January 2014, the semidiurnal barotropic tide in LS experienced a total of six spring-neap cycles (S1-S6, marked by red dashed lines in Fig. 5c).

\section{d. Vertical displacement}

Following Zhao et al. (2012), the vertical displacement in the upper layer is calculated through $\eta(z, t)=$ $[T(z, t)-\bar{T}(z, t)] / \bar{T}_{z}(z, t)$ based on the measurements from $T / S$ chains. Here, $T(z, t)$ denotes the temperature measurement, and $\bar{T}(z, t)$ is the background temperature calculated from $T(z, t)$ using a sliding 2.1-day (four semidiurnal periods) averaged window. The $\bar{T}_{z}(z, t)$ is the temperature gradient of $\bar{T}(z, t)$.

In the deep layer, $\eta(z, t)$ is calculated at the depth of CTDs. The variations in the temperature measurements caused by depth changes of CTDs, which are estimated by $\Delta z T_{z}(z)$, have been removed from temperature measurement. Here, $\Delta z$ is the depth changes of CTDs, and $T_{z}(z)$ is the averaged temperature gradient from CTD profiles in the northern SCS in winter, which were measured by SeaBird Electronics (SBE) 911/917 plus or SBE 25 from 2005 to 2012 (Yang et al. 2016). After the correction, the vertical displacement in the deep ocean is calculated via $\eta(z, t)=[T(z, t)-\bar{T}(z, t)] / T_{z}(z)$.

\section{e. Energy and flux of ITs}

In this study, the SIT energy and flux are computed in the same procedure as described in previous studies (Nash et al. 2005; Zhao et al. 2010, 2012). The depthintegrated horizontal kinetic energy (HKE) is calculated through 

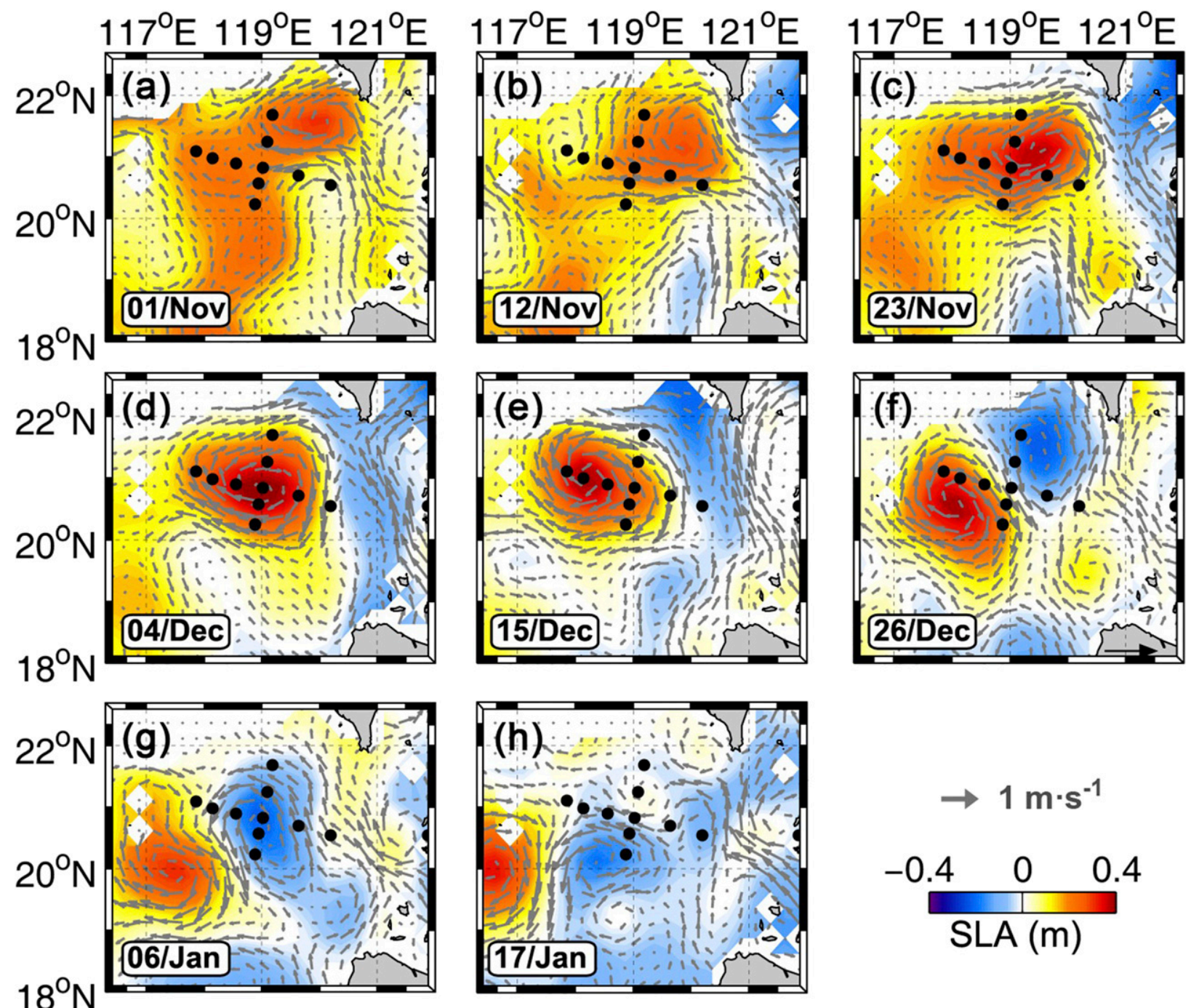

FIG. 3. The AVISO SLA (shading) and absolute geostrophic currents (vectors) in the experimental area during the winter of 2013/14. Black circles denote the mooring locations. Date is marked on each panel.

$\mathrm{HKE}=\int_{-H}^{0} \operatorname{hke}(z, t) d z=\frac{1}{2} \int_{-H}^{0}\left\langle\rho_{0}\left|\mathbf{u}^{\prime}(z, t)\right|^{2}\right\rangle d z$,

and available potential energy (APE) is calculated through $\mathrm{APE}=\int_{-H}^{0} \operatorname{ape}(z, t) d z=\frac{1}{2} \int_{-H}^{0}\left\langle\rho_{0} N^{2}(z, t) \eta^{2}(z, t)\right\rangle d z$

Here, $\mathbf{u}^{\prime}$ is the baroclinic current computed by subtracting the depth-averaged current at each time from the measured current; $\rho_{0}$ denotes the water density, $H$ denotes the water depth, and the angle brackets denote an average over one semidiurnal tidal cycle; $\operatorname{hke}(z, t)$ and ape $(z, t)$ are the nondepth-integrated horizontal kinetic and available potential energies, respectively. The $N^{2}(z, t)$ is the squared buoyancy frequency calculated from the potential density $\bar{\sigma}_{\theta}(z, t)$ smoothed by a 2.1-day sliding window.

A fourth-order Butterworth bandpass filter, with the central frequency at 1.93 cycles per day (cpd) and the cutoff frequencies of $[1.74,2.13] \mathrm{cpd}$, is performed to isolate the baroclinic velocity and displacement associated with the SIT. Then, total energy of the SIT is calculated through $E=\mathrm{HKE}+\mathrm{APE}$. For a progressive internal wave, the HKE to APE ratio (Zhao et al. 2010)

$$
r_{E}=\mathrm{HKE} / \mathrm{APE}=\left(\omega^{2}+f^{2}\right) /\left(\omega^{2}-f^{2}\right),
$$

where $\omega$ is the tidal frequency and $f$ is the inertial frequency. At M5 where the ADCP data are unable to resolve the internal tide signals, HKE of the SIT is obtained via $\mathrm{HKE}=r_{E}$ APE. At M6 where the mooring is 


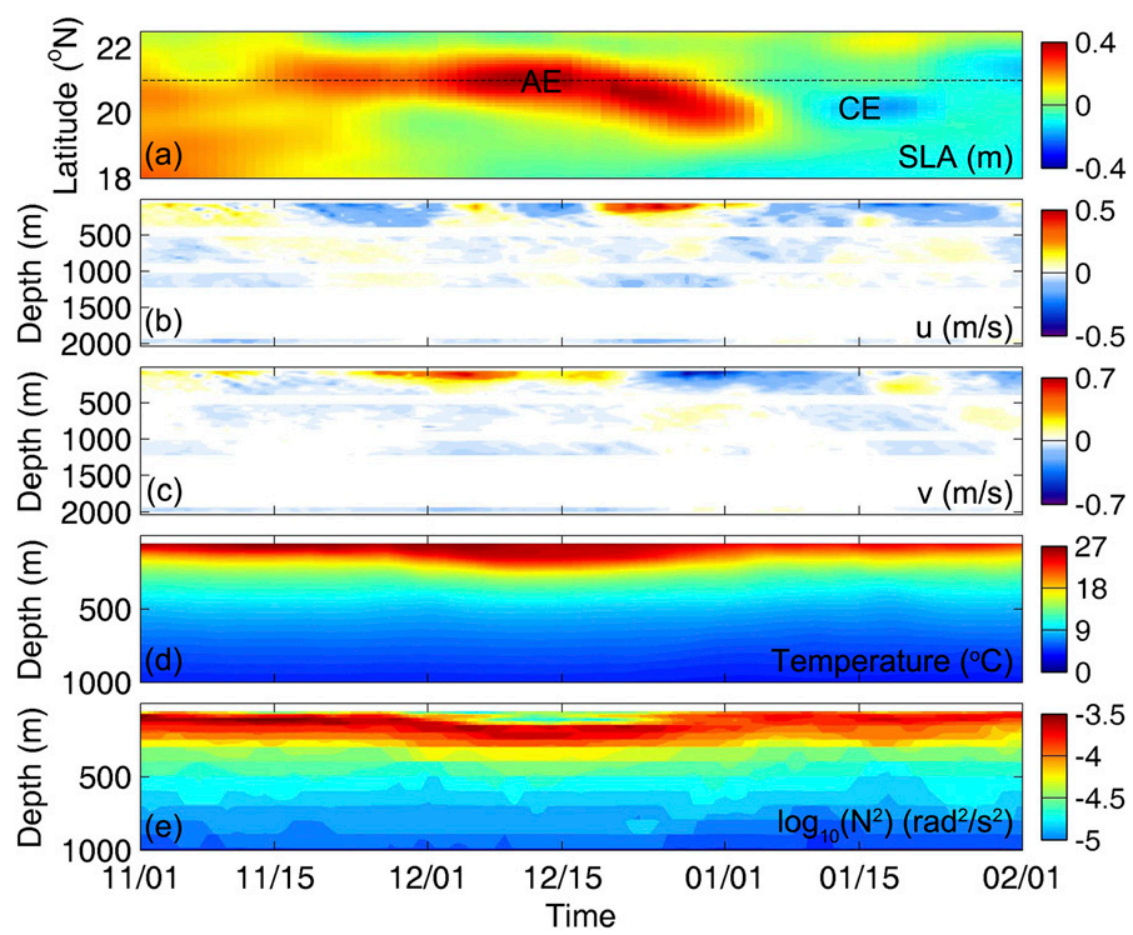

FIG. 4. (a) Hovmöller diagram of the SLA at $118.16^{\circ} \mathrm{E}$. The dashed line indicates the latitude of mooring M2. (b) Low-passed zonal and (c) meridional velocity at M2. (d) Temperature and (e) stratification at M2 smoothed by a 2.1-day sliding window.

lack of temperature measurement, APE is obtained via $\mathrm{APE}=\mathrm{HKE} / r_{E}$.

The depth-integrated SIT energy flux at the moorings is computed via

$$
\mathbf{F}=\int_{-H}^{0}\left\langle\mathbf{u}^{\prime}(z, t) p^{\prime}(z, t)\right\rangle d z
$$

Here, $p^{\prime}$ is the perturbation pressure that is computed from the displacement $\eta$ through (Nash et al. 2005; Zhao et al. 2010, 2012)

$$
p^{\prime}(z, t)=\rho_{0} \int_{-z}^{0} N^{2}(\hat{z}, t) \eta(\hat{z}, t) d \hat{z}-p_{\text {surf }}(t) .
$$

The $p_{\text {surf }}(t)$ is the surface perturbation pressure inferred from the baroclinic condition that the depth-integrated pressure perturbation is required to be zero:

$$
p_{\text {surf }}(t)=\rho_{0} \int_{-H}^{0} N^{2}(z, t) \eta(z, t) d z .
$$

The baroclinic velocity and displacement associated with ITs can be expressed by the superposition of discrete modes $\left[u^{\prime}, v^{\prime}\right]=\sum_{n=1}^{\infty}\left[\hat{u}_{n}, \hat{v}_{n}\right] \Pi_{n}(z)$ and $\eta(z, t)=$ $\sum_{n=1}^{\infty} \hat{\eta}_{n}(t) \Phi_{n}(z)$, respectively (Alford 2003; Nash et al. 2005; Zhao et al. 2010, 2012). Here, $\Pi(z)$ and $\Phi(z)$ are the normal mode of horizontal velocity and displacement, respectively. The $\hat{u}_{n}(t), \hat{v}_{n}(t)$, and $\hat{\eta}_{n}(t)$ are the time-varying $n$th modal magnitudes of zonal velocity, meridional velocity, and displacement, respectively. In this study, semidiurnal signals are decomposed into the first three modes at all moorings except M10. At M10, velocity is also decomposed into the first three modes, but displacement is only decomposed into two modes owing to the large gap of temperature measurement near the surface.

The Taylor-Goldstein equation in the presence of background shear and rotation

$$
\begin{aligned}
& {\left[f^{2}\left(U-c_{p}\right)-k^{2}\left(U-c_{p}\right)^{3}\right] \Phi^{\prime \prime}-f^{2} U^{\prime} \Phi^{\prime}} \\
& \quad+\left[k^{4}\left(U-c_{p}\right)^{3}+k^{2} U^{\prime \prime}\left(U-c_{p}\right)^{2}\right. \\
& \left.\quad-k^{2} N^{2}\left(U-c_{p}\right)\right] \Phi=0
\end{aligned}
$$

is obtained (see appendix A) from Eq. (8) in Frankignoul (1970). Here, the prime indicates the $z$ derivative, $c_{p}$ is the wave phase speed, $U(z)$ is the background current in the direction of wave propagation, and $k$ is the horizontal wave wavenumber. In Eq. (7), $k$ could be replaced with $\omega / c_{p}$, where $\omega$ is the frequency of the SIT $(1.93 \mathrm{cpd})$. With boundary conditions $\Phi(0)=\Phi(-H)=0$, Eq. (7) is numerically solved using the matrix method, and normal mode of displacement $\Phi(z)$ and phase speed $c_{p}$ 


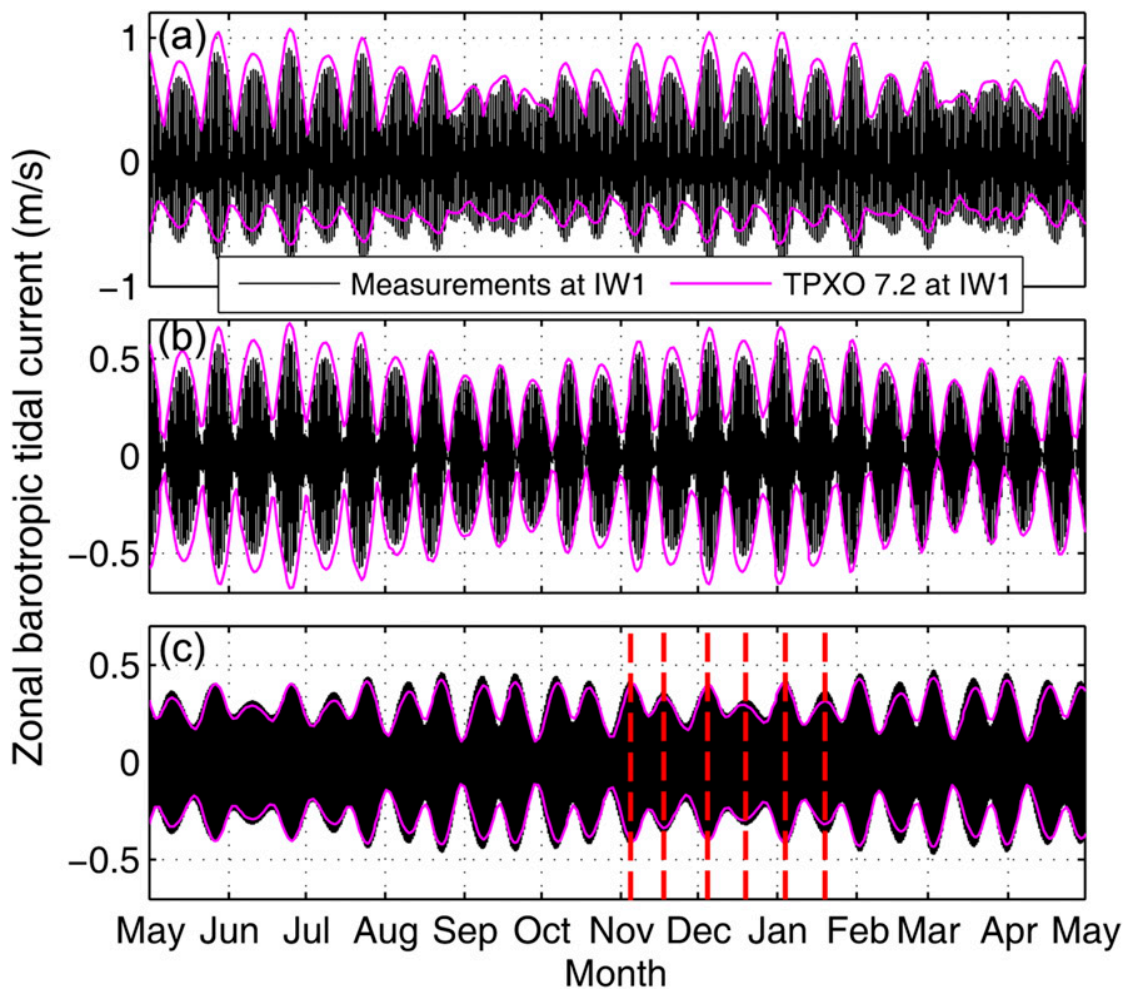

FIG. 5. Time series (black) of zonal (a) barotropic tidal current in LS measured at IW1 and their (b) diurnal and (c) semidiurnal components. Magenta curves denote the upper and lower envelopes of the predicted barotropic tidal current from TPXO 7.2. Red dashed lines in (c) mark the spring tide periods S1-S6 from November 2013 to January 2014 when the eddy pair crossed the northern SCS.

of the SIT are derived (see appendix A). The structure of $\Pi(z)$ is obtained from the derivative of $\Phi(z)$ with respect to $z$.

Because of the migrating eddies, the stratification and background current in the northern SCS varied much with time. In this study, the Taylor-Goldstein equation is solved with time-varying stratification and background current. Figure 6 presents the current ellipses for the SIT in the upper $1000 \mathrm{~m}$ at mooring M2 during the spring tide periods S1-S6, which are obtained from the harmonic analysis of baroclinic currents in sliding 2-day windows using the T_TIDE toolbox (Pawlowicz et al. 2002). The inclination of current ellipses of the SIT varied among different spring tide periods, indicating that the propagation direction of the SIT changed possibly because of the moving eddies. Here, the propagation direction of the SIT is estimated to be the average (black arrows in Fig. 6) of the inclination of current ellipses in the upper $800 \mathrm{~m}$ weighted by the length of the semimajor axes. Then, the background current in the propagation direction of the SIT is computed. As shown in Fig. 6, the estimated propagation direction of the SIT (black arrows) at M2 agreed well with the orientation of the depth-integrated SIT energy flux vectors (red and cyan arrows) during the eddy period, indicating that it is appropriate here to estimate the propagation direction of the SIT from the weighted average of the inclination of SIT current ellipses.

At M6 where the mooring is lack of $T / S$ data, the magnitude of depth-integrated SIT energy flux is obtained from the computed energy flux at M3 and M9 through linear interpolation. The orientation of energy flux at M6 is estimated to be the weighted average of the inclination of SIT current ellipses in the upper $800 \mathrm{~m}$. At M5 where the ADCP data are unable to resolve IT signals, both the magnitude and orientation of depth-integrated SIT energy flux are obtained from the computation results at M4 and M6 through linear interpolation. The errors in the SIT energy and flux computations due to the vertical gaps in the measurements are assessed in appendix B.

\section{f. Phase speed of the mode-1 SIT between two moorings}

In the along-beam direction, phase speed of the mode-1 SIT between two moorings is also computed from observations through (Huang et al. 2017) 

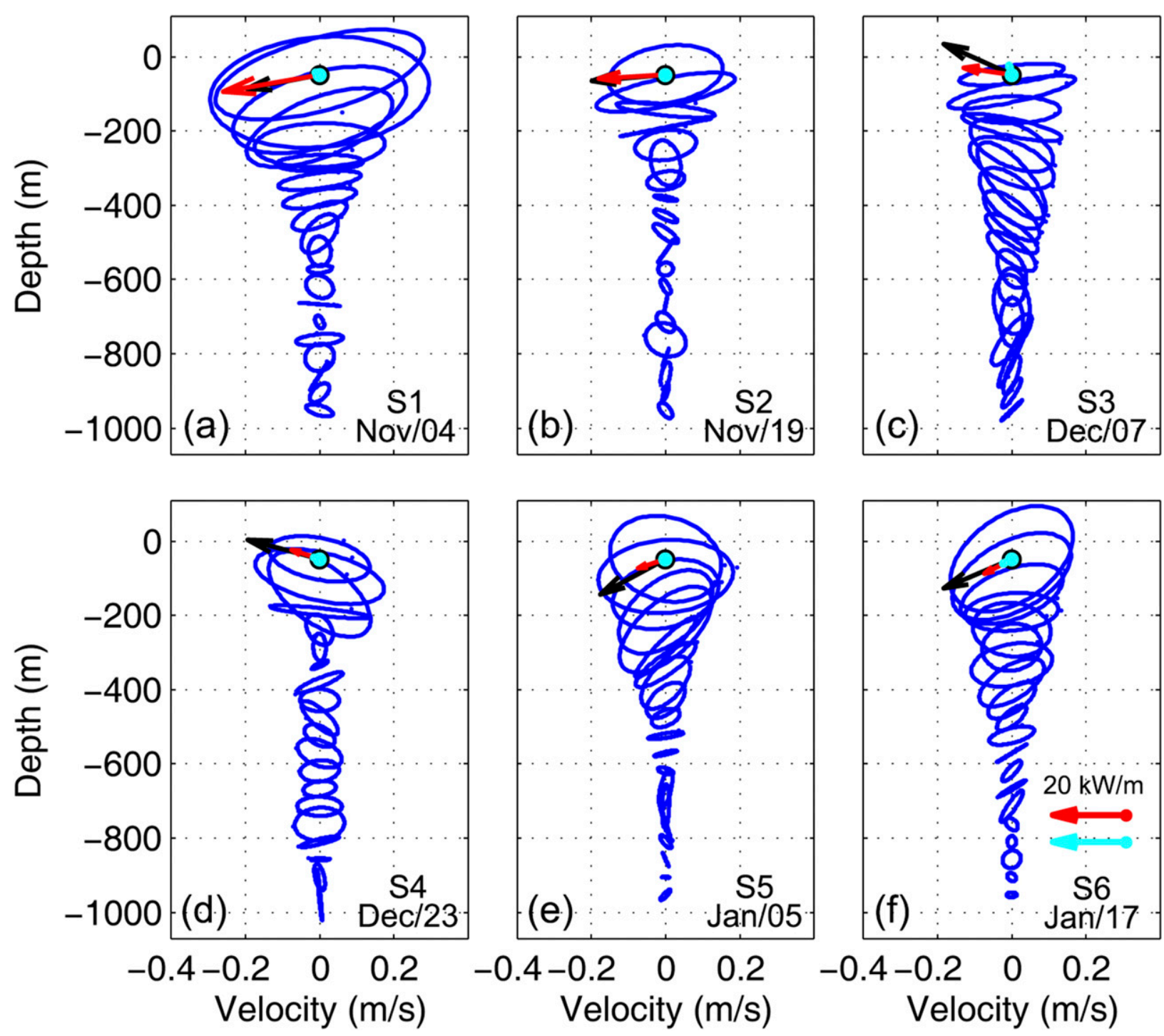

FIG. 6. Current ellipses for the SIT at mooring M2 during the spring periods S1-S6. The black arrows denote the weighted average of the inclination of current ellipses of the SIT in the upper $800 \mathrm{~m}$. The red and cyan arrows denote the computed depth-integrated mode- 1 and mode-2 SIT energy flux vectors, respectively.

$$
c_{p}=D \cos (\theta) / T_{m}
$$

where $D$ and $T_{m}$ denote the distance and traveling time of the SIT between two moorings in the along-beam direction, respectively, and $\theta$ is the angle between the mooring lie and the mode- 1 SIT energy flux vector. Time $T_{m}$ is obtained based on the maximum cross correlation of the mode-1 SIT currents in the upper layer between two moorings.

\section{g. Pattern of the mode-1 SIT wave crest}

The pattern of the mode-1 SIT wave crest is computed using the following procedure from the bandpassfiltered semidiurnal signals at the cross-beam-direction moorings. We first obtained the arrival time of the mode-1 SIT wave crest at the cross-beam-direction moorings. Then, we computed the differences in the arrival time $\Delta T$ of the mode- 1 SIT wave crest at M4-M7 to that at the northernmost mooring M8 (the snapshot of the SIT wave crest pattern was obtained at the time $T_{\mathrm{M} 8}$, when the SIT wave crest arrived at M8). Third, we computed the distance from the location of the mode-1 SIT wave crest to moorings M4-M7 at the time $T_{\mathrm{M} 8}$, respectively, through $\Delta d=\Delta T c_{p}$. Fourth, we traced the location of the mode- 1 SIT wave crest that would arrive at M4-M7 based on the $\Delta d$ and the orientation of energy flux. Finally, we obtained the mode-1 SIT wave crest pattern after smoothing 


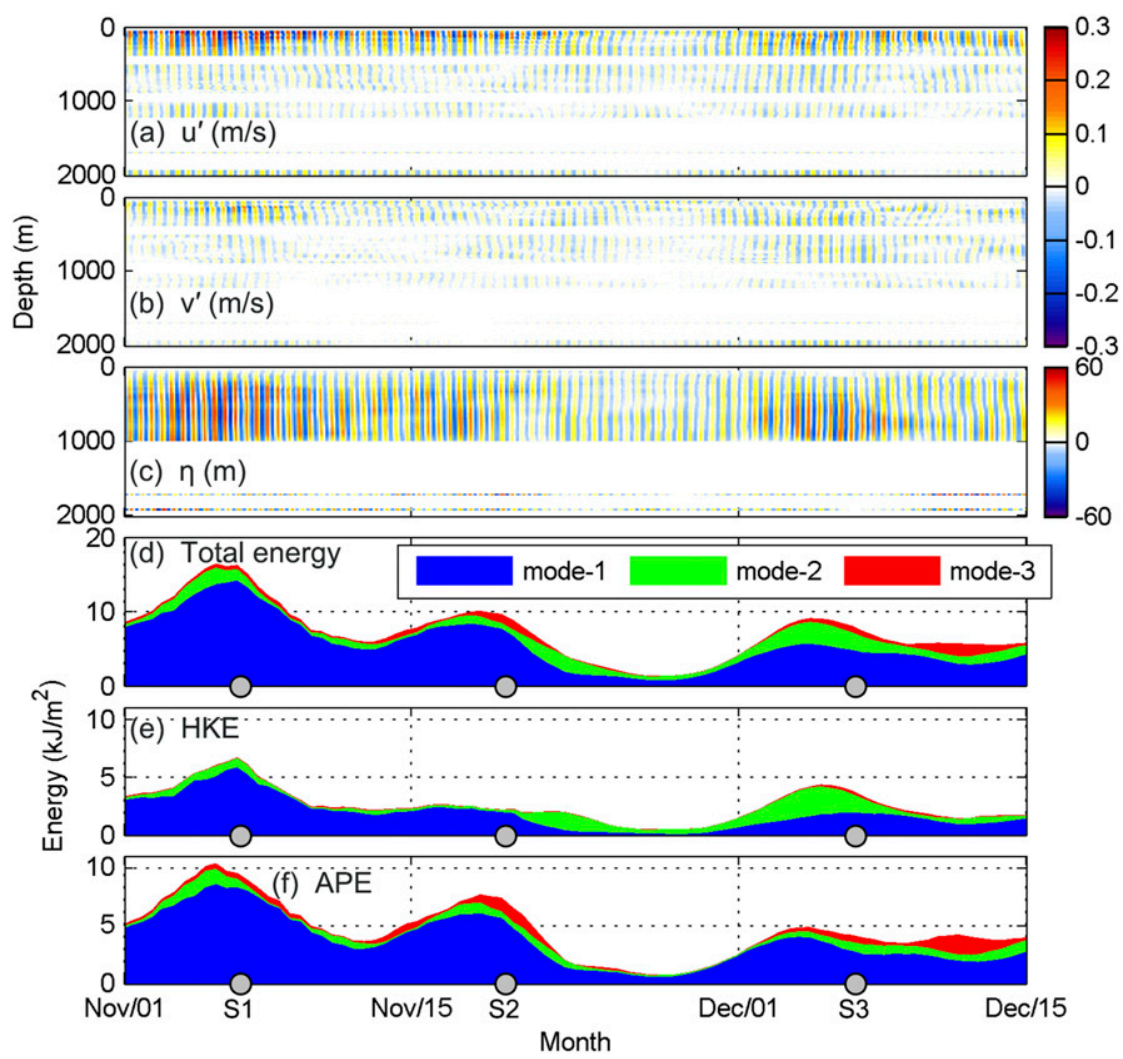

FIG. 7. The SIT at mooring M2 between 1 Nov and 15 Dec. (a)-(c) Zonal, meridional baroclinic velocity and vertical displacement associated with the SIT. (d)-(f) Depth-integrated total energy, HKE, and APE of the lowest three modes of SIT. The gray dots denote the spring tide periods $\mathrm{S} 1-\mathrm{S} 3$.

the location of the mode-1 SIT wave crest with cubic spline interpolation.

\section{Results}

\section{a. Mode-1 SIT energy modulated by the eddies}

Figures $7 \mathrm{a}-\mathrm{c}$ present the computed zonal, meridional baroclinic velocities and vertical displacement associated with the SIT, respectively, at mooring M2 between 1 November and 15 December. Strong baroclinic velocities associated with the SIT can be clearly seen near the sea surface, and they weaken remarkably as they approach $800 \mathrm{~m}$, which is the depth of the mode- 1 node. The shaded areas in Figs. $7 \mathrm{~d}-\mathrm{f}$ denote the cycle-mean depth-integrated total energy, HKE, and APE of the lowest three modes SIT at mooring M2, respectively. During the eddy period, the magnitude of the SIT energy experienced significant changes among different spring tide periods. For an example, the peak of the SIT energy during the spring tide period S1 was twice that during S3.

Gray shading areas in the left panels of Fig. 8 present the mode-1 SIT energy at the along-beam-direction moorings. Near the generation sites in LS at M10, the mode-1 SIT energy varied significantly from one spring tide period to another, probably owing to the varying SIT generation in LS, which was irrelevant to the eddy pair. To qualitatively evaluate the changes of the mode1 SIT energy associated with the eddy pair, the following processing is done so as to exclude the influences of the varying SIT generation in LS. First, the ratios of the depth-integrated mode-1 SIT energy at M1-M8 to that at M9 near LS [owing to the relatively large errors in the APE computation at M10 (see appendix B), the depthintegrated mode-1 SIT energy at M9 instead of M10 is chosen here to provide a reference] $r_{n}$ are calculated during the "no eddy" periods (20 January-4 March and 3 May-2 June). Second, a no-eddy reference (red curves in Fig. 8) for the depth-integrated mode-1 SIT energy at M1-M8 is obtained, via multiplying the mode-1 SIT energy at M9 by $r_{n}$. Through comparing blue curves to red curves in Figs. 8a-f, we can see that the depth-integrated mode-1 SIT energy at the along-beam-direction moorings generally showed concurrent responses to the eddies during the eddy period. An obvious example is the 

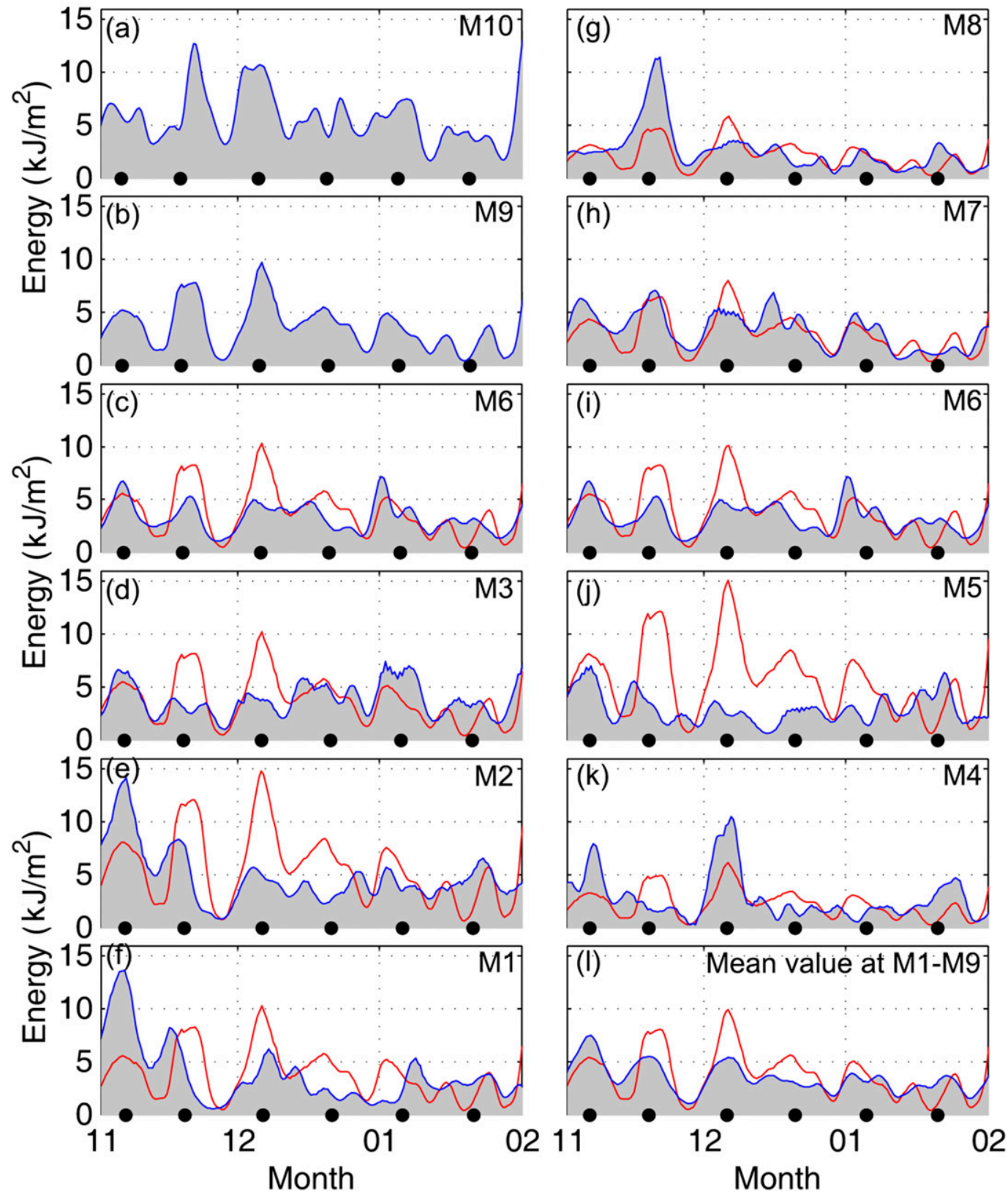

FIG. 8. (a)-(f) Depth-integrated mode-1 SIT energy at the line of moorings in the along-beam direction (blue curves or gray shaded region). The red curves denote the no-eddy reference. The black dots denote the spring tide periods S1-S6. (g)-(k) As in (a)-(f), but at the line of moorings in the cross-beam direction. (l) The mean depthintegrated mode-1 SIT energy at moorings M1-M9.

decreased depth-integrated mode-1 SIT energy during the spring tide periods S2 and S3.

Gray shaded areas in Figs. 8g-k exhibit the observed depth-integrated mode-1 SIT energy at the cross-beamdirection moorings. Comparisons between the observed mode-1 SIT energy and the no-eddy reference (red curves) show that the responses of the depth-integrated mode-1 SIT energy to the eddies were nonmonotonic at the cross-beam-direction moorings. Especially, during the period S2, the depth-integrated mode- 1 SIT energy increased in the north part of the northern SCS at M7 but decreased in the south part of the northern SCS at M4-M6; during the period S3, the depth-integrated mode-1 SIT energy decreased at M7 and M8 but increased at M4. These results show that in the north and south parts of the northern SCS, the responses of the 


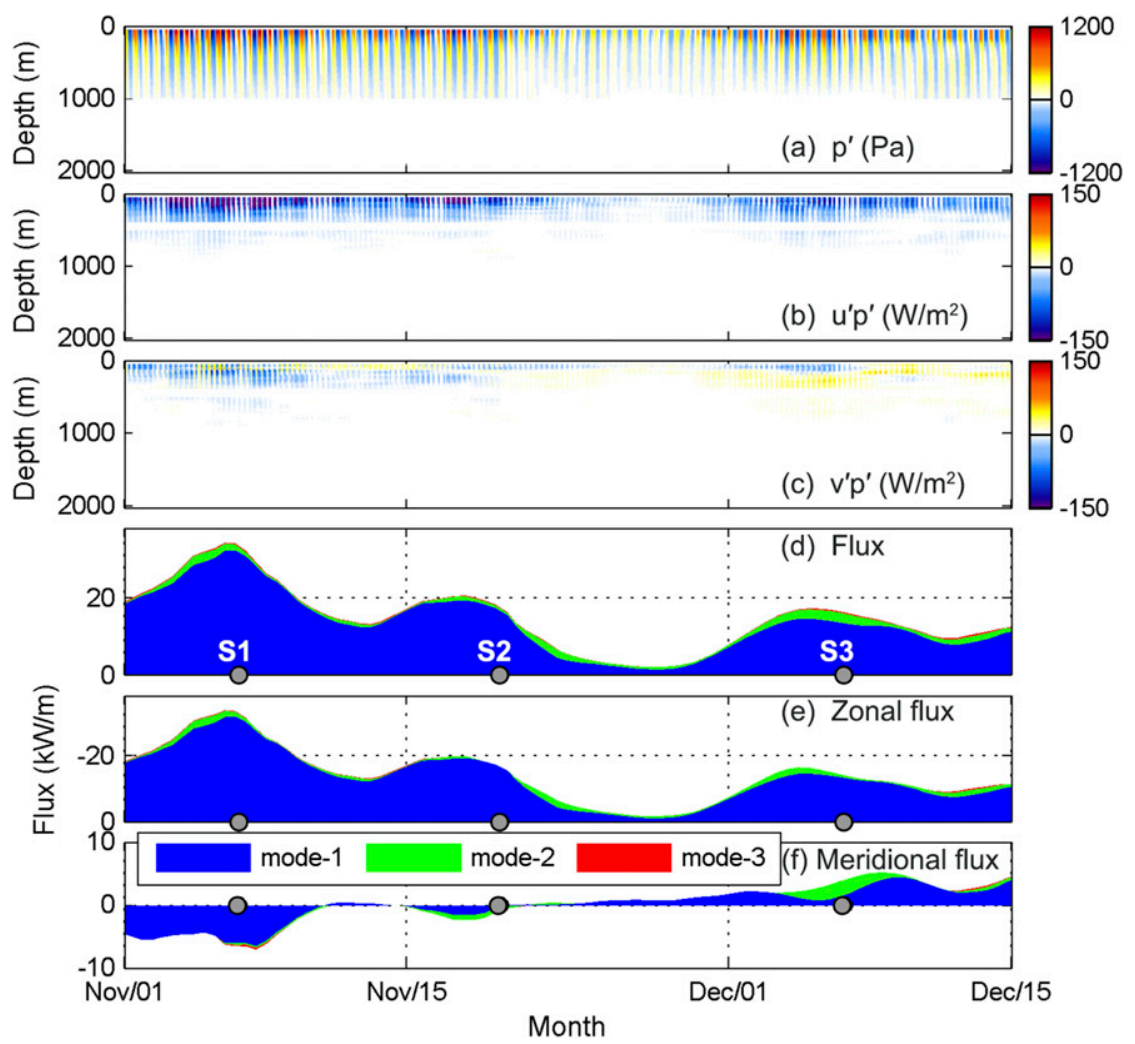

FIG. 9. (a) Pressure perturbation, (b) zonal, and (c) meridional energy flux of the SIT at mooring M2. (d)-(f) Depth-integrated total, zonal, and meridional energy flux of the lowest three modes SIT at mooring M2. The gray dots denote the spring tide periods S1-S3.

depth-integrated mode-1 SIT energy to the eddies were out of phase during the two spring tide periods.

During the whole eddy period, the most remarkable drop of $74 \%$ in the depth-integrated mode-1 SIT energy occurred at M5 during the period S3, when the mooring was on the southern boundary of the AE. The largest increase of $119 \%$ in the depth-integrated mode- 1 SIT energy happened at the northernmost mooring M8 during the period $\mathrm{S} 2$, when the mooring was covered by the northern portion of the AE.

\section{b. Mode-1 SIT energy flux modulated by the eddies}

The computed pressure perturbation, east-westward energy flux $u^{\prime} p^{\prime}$, and north-southward energy flux $v^{\prime} p^{\prime}$ associated with the SIT at mooring M 2 between 1 November and 15 December are shown in Figs. 9a-c. The SIT energy flux is concentrated near the sea surface, suggesting the dominance of the mode- 1 signal. In the zonal direction, the energy flux is always dominated by the westward component. In the meridional direction, the southward component of energy flux was dominant during the period S1, but the reverse was true during the period S3.
Understanding the manner in which the SIT energy propagates inside the eddies is important to determining its horizontal distribution and dissipation. Figure 10 presents the cycle-mean depth-integrated energy flux vectors of the mode-1 SIT at the moorings. Because of the propagation of the eddy pair, the energy flux of the mode-1 SIT in the northern SCS exhibited different spatial patterns. During the period S1, the main mode-1 SIT energy path was located to the south of M8 toward Dongsha Island. When the AE moved closer to the mooring array during the period S2, most of the mode-1 SIT energy was refracted away from Dongsha Island toward the north part of the northern SCS. The main propagation path of the mode-1 SIT energy reappeared in the region to the south of M8 during the period S3 when the AE core was located near the mooring array center. When the AE core was located to the west of the mooring array center during the period S4, the propagation path of the mode-1 SIT energy was converged around the line of moorings in the along-beam direction. When the CE influenced the mooring array during the period S5, most of the mode-1 SIT energy was refracted away 

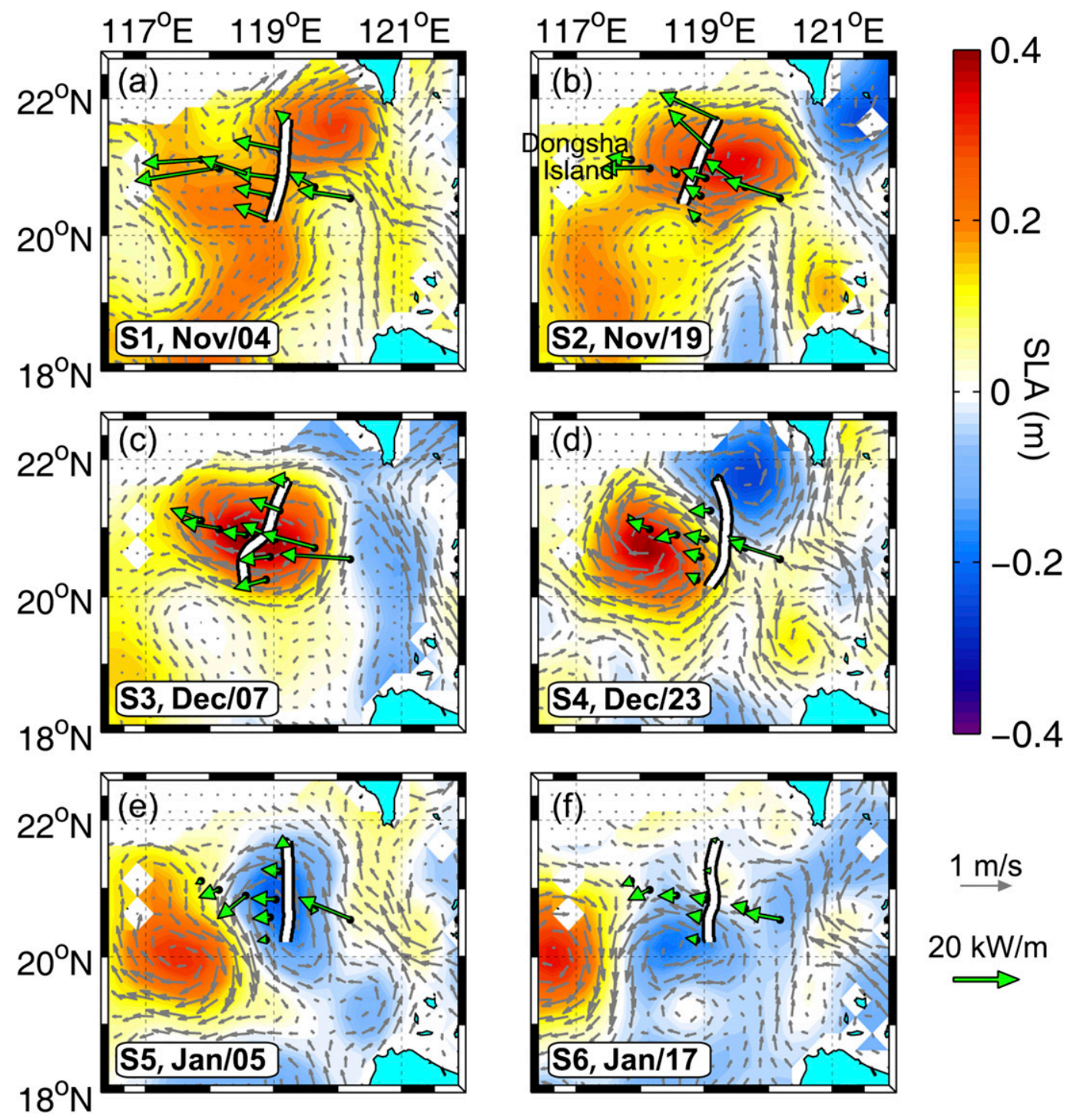

FIG. 10. Depth-integrated energy flux vector of the mode-1 SIT (green arrows). Gray arrows denote the absolute geostrophic currents from AVISO. The white curves denote the pattern of the mode- 1 SIT wave crest.

from Dongsha Island toward the south part of the northern SCS.

During the period S2, most of the mode-1 SIT energy was refracted by the AE toward the north part of the northern SCS (Fig. 10b), and a "desert" region of the SIT energy was formed around the mooring M3. Hence, the mode-1 SIT energy at downstream moorings in the along-beam direction (M1-M3) decreased concurrently during this period (Figs. 8c-f). The opposite changing tendencies of the mode- 1 SIT energy in the south (Figs. 8j,k) and north (Figs. 8g,h) parts of the northern SCS at the cross-beam direction mooring during the period S2 were also mainly resulted from the northward refraction of energy flux by the AE.

\section{c. Phase speed of mode-1 SIT modulated by the eddies}

Bold black curves in Fig. 11 show that the observed phase speed of the mode-1 SIT in the northern SCS varied significantly during the eddy period. It generally reached a peak inside the southern portion of the AE but experienced a low within the southern portion of the CE. For example, the mode- 1 SIT from M10 to M9 propagated at a speed of $3.63 \mathrm{~m} \mathrm{~s}^{-1}$ inside the southern portion of the $\mathrm{AE}$ on November 20, which was almost $0.7 \mathrm{~m} \mathrm{~s}^{-1}$ faster than the reference $c_{p}$ (gray dashed line) obtained through solving the Taylor-Goldstein equation with the winter WOA stratification and zero background current; it propagated at a speed of $2.58 \mathrm{~m} \mathrm{~s}^{-1}$ 

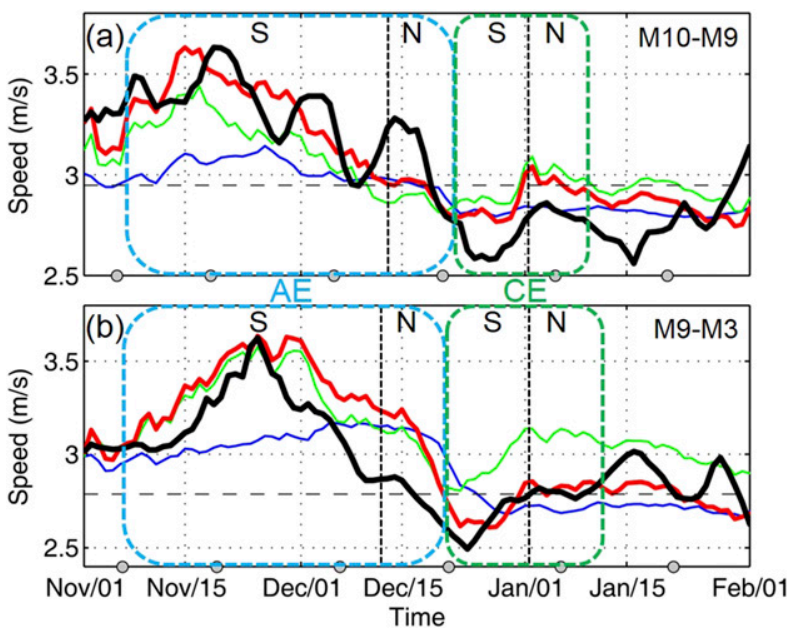

FIG. 11. (a) Observed mean $c_{p}$ of the mode-1 SIT between M10 and M9 (black). The red curve denotes the theoretical $c_{p}$ through solving the Taylor-Goldstein equation. Blue and green curves denote the theoretical $c_{p}$ when only considering time-varying stratification and background current, respectively. The gray dashed line denotes the reference $c_{p}$ that was computed from the mean winter WOA stratification with zero background current. Cyan and green rectangles mark the duration periods of the $\mathrm{AE}$ and the CE, respectively. The vertical dashed lines are used to separate the southern and northern portions of the two eddies. The gray dots denote the spring tide periods S1-S6. (b) As in (a), but between M9 and M3.

inside the southern portion of the $\mathrm{CE}$ on December 27 , which was $\sim 0.4 \mathrm{~m} \mathrm{~s}^{-1}$ lower than the reference $c_{p}$. Additionally, inside the AE, the mode-1 SIT propagated faster within its southern portion than within its northern portion. For an example, its mean phase speed between M10 and M9 was 3.33 and $3.13 \mathrm{~m} \mathrm{~s}^{-1}$, respectively, within the AE's southern and northern portions. The reverse occurred as the mode- 1 SIT encountered the $\mathrm{CE}$, when it propagated more slowly within the southern portion of the $\mathrm{CE}$ than within its northern portion.

The theoretical $c_{p}$ (thick red curves in Fig. 11) derived through solving the Taylor-Goldstein equation with timevarying stratification and background current shows comparable magnitudes and similar changing trends to the observed $c_{p}$. However, when only considering the time-varying stratification or background current, there exists evident deviation between the theoretical $c_{p}$ (green and blue curves) and the observed $c_{p}$. When propagating through the southern portion of the $\mathrm{AE}$, both the eddy-induced background current and stratification anomalies tended to accelerate the phase speed of the mode-1 SIT, and thus it reached a peak. On the contrary, as propagating through the southern portion of the $\mathrm{CE}$, both the eddy-induced background current and stratification anomalies decelerated the phase speed of the mode-1 SIT, and hence it reached a trough.

\section{d. Distortion of wave crest of mode-1 SIT by the eddies}

The thick white curves in Fig. 10 show the pattern of the mode- 1 SIT wave crest computed based on the measurements at the cross-beam-direction moorings. It can be seen that the mode- 1 SIT wave crest showed different patterns owing to the migrating eddies. Notably, during the period S2, the mode- 1 SIT wave crest inside the AE core (defined as the part of the eddy within the circle of maximum rotational velocity) was clockwise rotated by $6^{\circ}$ in comparison with the climatological result (toward northwest with a direction of $165^{\circ}$ from due east) in Zhao (2014). During the period S3, the northern part of the mode-1 SIT wave crest inside the AE core was also clockwise rotated, while the southern part of the wave crest, which was located on the southern boundary of the AE, exhibited a convex shape. During the period S4, the mode-1 SIT wave crest showed a concave shape, because its central portion, which was located on the connection zone between the two eddies, fell behind its two edges. During the period S5, the mode-1 SIT wave crest inside the CE core was mainly in the north-south direction, indicating that it had been counterclockwise rotated by the $\mathrm{AE}$ by $15^{\circ}$ in comparison with the climatological result in Zhao (2014). During the period S6, a convex shape was seen in the southern part of the mode-1 SIT wave crest, which was on the northern boundary of the CE.

From Fig. 10, we can see that the mode-1 SIT wave crest within the AE (CE) core were generally clockwise rotated (counterclockwise rotated). This was reasonable because the mode-1 SIT propagated faster (slower) within the AE's (CE's) southern portion than within its northern portion (section 3c). Moreover, on the southern (northern) boundary of the AE (CE), the mode-1 wave crest exhibited a convex pattern, because the mode-1 SIT, which was accompanied by the maximum westward eddy current, propagated faster than its two sides. The reverse was true on the northern (southern) boundary of the AE (CE), where the mode-1 SIT wave crest showed a concave pattern.

Figures $10 \mathrm{~b}$ and $10 \mathrm{e}$ show that, as a result of the rotated wave crest by the eddies, the mode-1 SIT energy was refracted northward and southward during the periods S2 and S5, respectively, resulting in the changes in the mode- 1 SIT energy observed at the moorings. In addition, the distortion of the mode- 1 SIT wave crest, which possibly led to the convergence/divergence of the SIT energy, might also result in the variations in the observed mode-1 SIT energy. By calculating the ratio of the length of actual wave crest to that of idealized wave crest (a line toward northwest with a direction of $165^{\circ}$ 
from due east), we evaluated the changes in the mode-1 SIT energy density associated with the distortion of the mode- 1 SIT wave crest. The mode- 1 SIT crest exhibited a concave shape during the period S4. During this period, the increased length of the wave crest would give rise to an average of $12 \%$ drop in the mode- 1 SIT energy at M5-M7. During the periods S3 and S6, the southern part of the wave crest showed a convex shape, and the increased wave crest length accounted for $27 \%$ and $6 \%$ drop in the mode- 1 SIT energy at M5, respectively.

\section{e. Interference of mode-1 SIT during the eddy period}

For a free progressive wave, the ratio of HKE to APE $r_{E}$ is a constant depending on the tidal frequency $\omega$ and local inertial frequency $f$, as suggested by Eq. (3). In our study region, the theoretical $r_{E}$ for the free-propagating SIT ranges within [1.29, 1.34]. However, when two waves travel in opposite directions, the interference happens and $r_{E}$ deviates from the theoretical value, which varies from zero to infinity depending on the incident wave phases (Martini et al. 2007).

The observed $r_{E}$ of the mode-1 SIT is shown in Fig. 12. At the westernmost mooring M1, the observed $r_{E}$ agreed well with the free-wave value of 1.32 (red dashed line in Fig. 12a) in early November during the period S1. In contrast to the free-wave value, the observed APE at M1 exceeded HKE when the AE approached during the periods S2-S4. When the CE approached during the period S6, HKE was larger than APE and the observed HKE-to-APE ratio was much larger than the free-wave value. The partitioning between HKE and APE at M1 varied when the eddies approached, implying the interference pattern of the SIT during the eddy period. At other moorings in the array, the mode-1 HKE/APE ratio also varied significantly during the eddy period. The deviation of $r_{E}$ from the free-wave values suggests that interference of the SIT might happen at those moorings. As can be seen in Fig. 10, the SIT energy flux in the northern SCS was no longer uniform but multidirectional because of the eddy pair. Hence, at a given site, the SIT energy might come from several directions when the eddies happened, resulting in the interference of the SITs. Through performing numerical investigations, Ponte and Klein (2015) also found that the IT's signature on sea level formed complex interference patterns in the strong eddy field.

\section{f. Impacts of eddies on the formation of ISWs}

ISWs in the northern SCS can be classified into two types: type-A ISWs, which appear about the same time each day with larger amplitudes, and type-B ISWs, which appear about one hour later each day with smaller

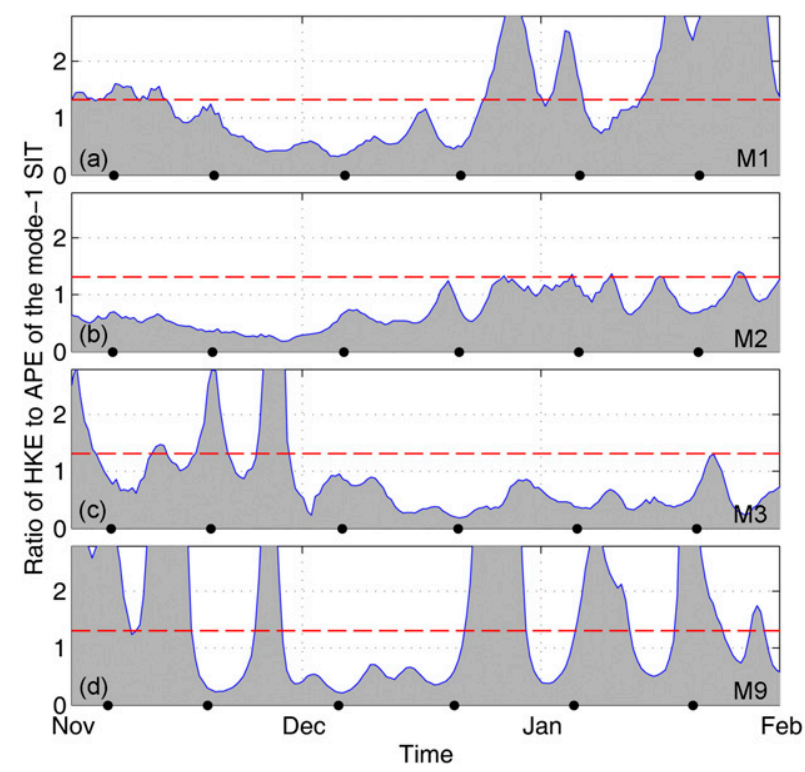

FIG. 12. The ratio of HKE to APE of the mode-1 SIT at the line of moorings in the along-beam direction. The red dashed lines denote the theoretical value. The black dots denote the spring tide periods S1-S6.

amplitudes (Alford et al. 2010; Huang et al. 2014; Ramp et al. 2010). In Huang et al. (2017), we have demonstrated that the eddy pair significantly impacted the propagation of type-A ISWs. Type-B ISWs generally evolve from the SIT via nonlinear steepening process (Helfrich and Grimshaw 2008; Li and Farmer 2011) during its propagation in the northern SCS. Here, we further examine the variations of type-B ISWs in the northern SCS due to the interactions between the SIT and mesoscale eddies.

The left and right panels in Fig. 13 present a one-day segment of measurement at the cross-beam-direction moorings (M4-M8) during the eddy and no-eddy periods, respectively. In the absence of eddies on 2 February, no ISW was observed in the north part of the northern SCS at M8. However, in the presence of the AE during the period $\mathrm{S} 2$ on 20 November, both type-A and type-B ISWs were evident at M8. The detectable type-A ISW at M8 during this period was possibly due to the broadening of the wave crest caused by the AE [see Fig. 8c in Huang et al. (2017)]. On the other hand, the evident type-B ISWs at M8 during this period was attributed to the northward refraction of the SIT energy by the AE (Fig. 10b). The relatively weak type-B ISWs in the south part of the northern SCS at M4 and M5 on 20 November were consistent with the northward refraction of the SIT energy.

Figure 14 presents the amplitude of type-B ISWs at the cross-beam-direction moorings between November 


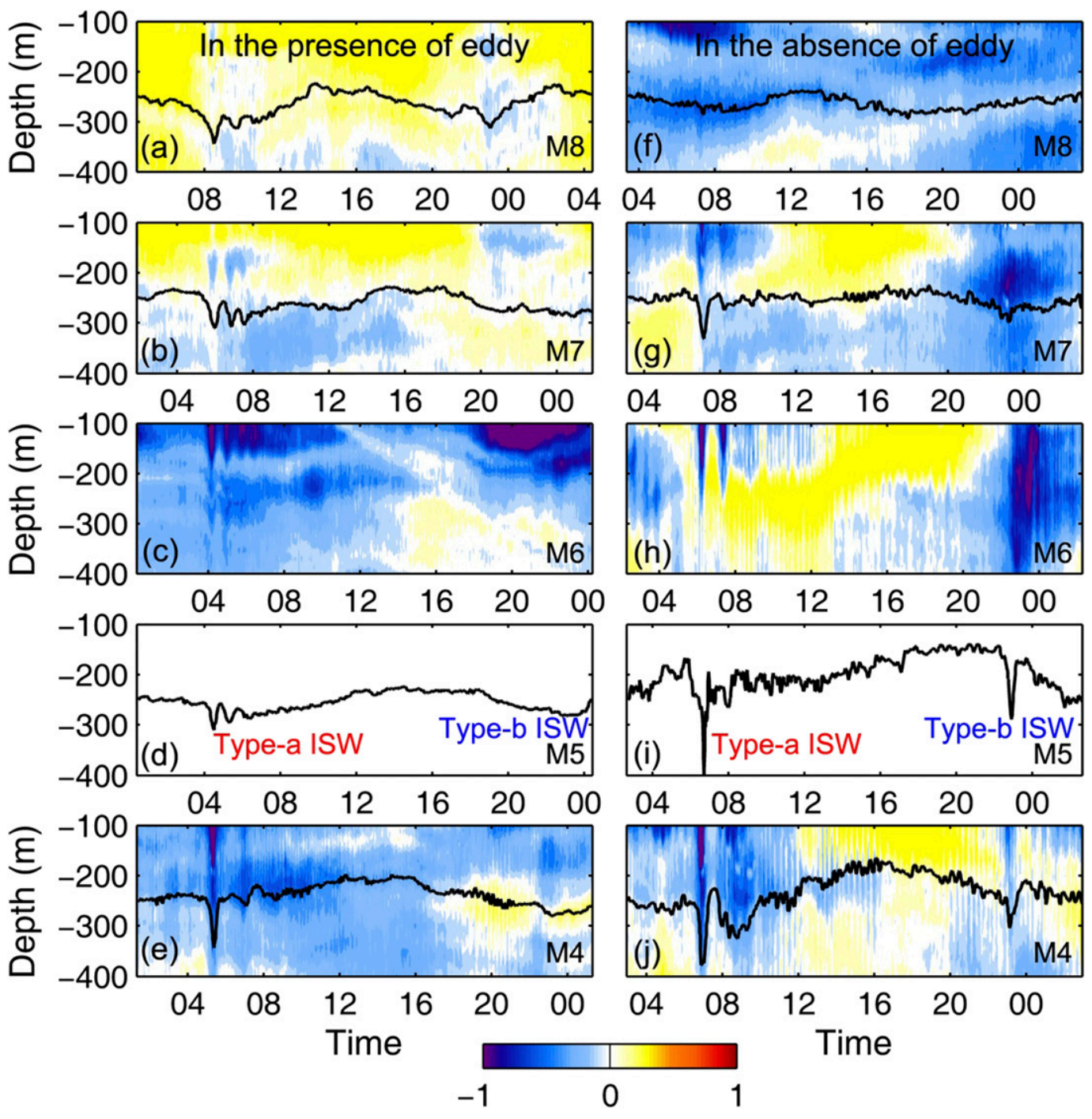

FIG. 13. (left) A one-day segment of zonal velocity measurement $\left(\mathrm{m} \mathrm{s}^{-1}\right)$ at the line of moorings in the cross-beam direction during the eddy period on 20 Nov 2013. Black curves denote the isothermal displacement at the 250-m depth. (right) As in the left panels, but during the no-eddy period on 2 Feb 2014.

2013 and January 2014. During the periods S1-S4 when the AE occurred, the changing tendencies of amplitude of type-B ISWs in the north part of the northern SCS at M8 were generally out of phase to those in the south part of the northern SCS at M4 and M5. This was due to the modulations of eddies in the spatial distribution of the SIT energy (Figs. 8g-k). During the period S5 in early January, both type-B ISWs in the south and north parts of the northern SCS were relatively strong. We note that the cross-beam-direction moorings were covered by the $\mathrm{CE}$ during this period, which resulted in thermocline shoaling (Fig. 14e, marked by the red arrow) from M10 near LS to the cross-beam-direction moorings. The thermocline shoaling is believed to be favorable for the formation of ISWs (Buijsman et al. 2010;
Zheng et al. 2007). Hence, a shoaling thermocline from LS to the northern SCS associated with the CE possibly accounted for the formation of the relatively large typeB ISWs during the period S5.

\section{Discussion}

In comparison with the mode-1 wave, the shear of higher-mode waves is relatively large, which can trigger turbulent mixing more effectively. Figure 15 presents the depth-integrated mode- 2 and mode- 3 SIT energy at the moorings during the eddy period. Generally, the higher-mode SITs are considered to be generated in LS and dissipate in the region around LS. However, during the periods S2-S4 when the eddy pair happened, the 

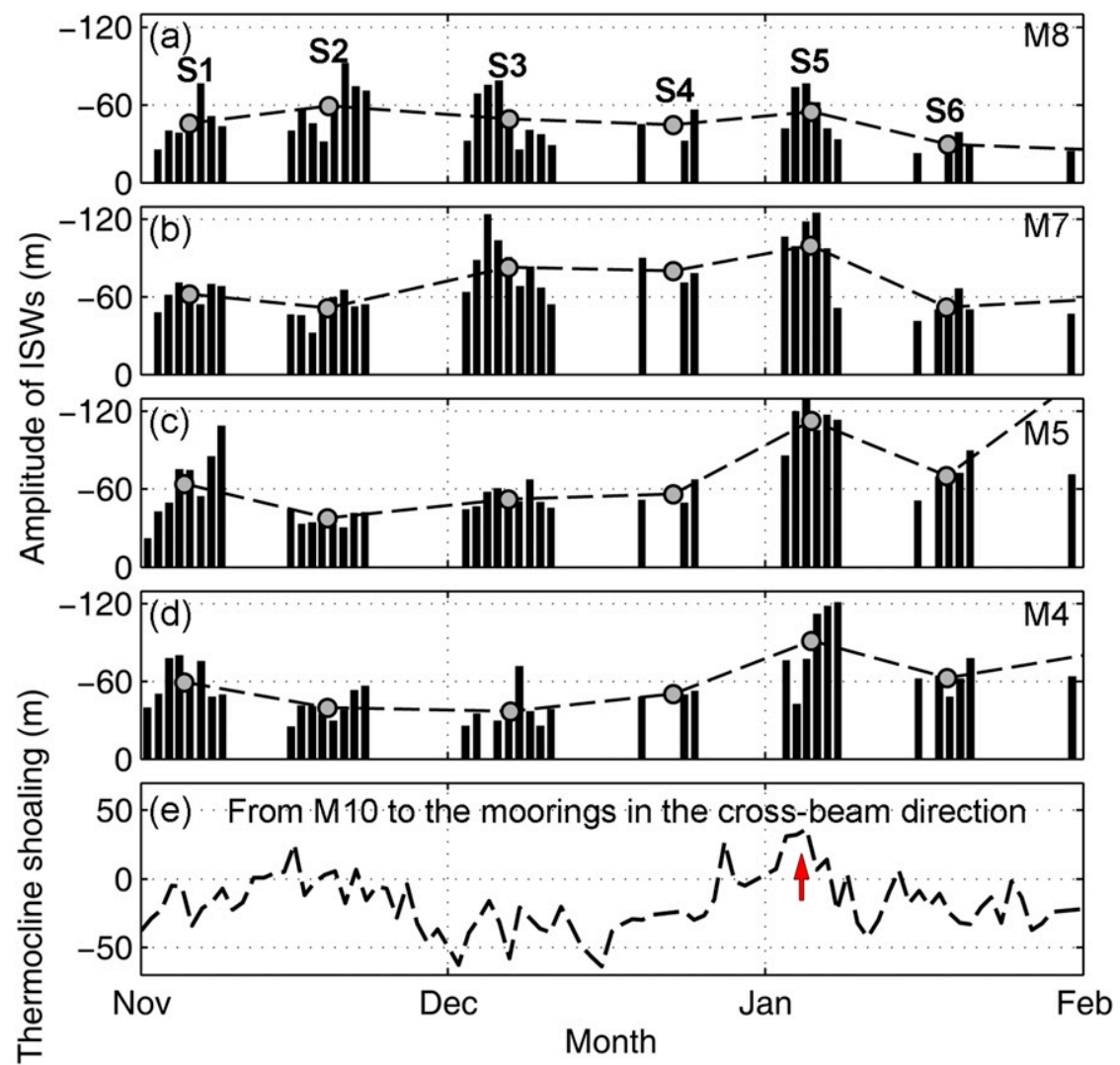

FIG. 14. (a)-(d) Amplitude of type-B ISWs measured at the line of moorings in the crossbeam direction. The gray dots denote the mean amplitude of type-B ISWs over each springneap tidal cycle. (e) Thermocline depth variations from M10 to the moorings in the cross-beam direction, computed from the $12^{\circ} \mathrm{C}$ isotherm depth.

mode-2 SIT was found to be energetic inside the northern SCS at M3, M4, M6, and M9. Especially, during the period S3, the magnitude of depth-integrated mode2 SIT energy at the four moorings was more than 5 times as large as that during the period S1 when those moorings were outside of the eddies.

Using a mathematical model, Lelong and Riley (1991) demonstrated that wave-wave-vortex triad interactions could be found between two equal-frequency waves and one vortical mode and the vortex facilitated energy transfer between two wave modes by acting as a catalyst. In numerical simulations, Dunphy and Lamb (2014) found that, a mode- 1 baroclinic eddy could result in the scattering of energy from the incident mode- 1 ITs to modes 2 and higher. During the periods S2-S4, the mean depth-integrated mode-1 SIT energy (gray shaded area in Fig. 81) at moorings M1-M9 was obviously smaller than the reference (red curve). The decreased mode- 1 SIT energy and increased mode- 2 SIT energy during the periods S2-S4 suggested that, through interacting with mesoscale eddies, the mode- 1
SIT was a possible energy source for the intensified higher-mode SITs.

\section{Summary}

In this study, the role of mesoscale eddies in modulating the semidiurnal internal tide (SIT) in the northern SCS is investigated using the data from a cross-shaped mooring array consisting of 11 moorings. From November 2013 to January 2014, an energetic eddy pair consisting of one $\mathrm{AE}$ and one $\mathrm{CE}$ crossed the westward SIT beam originating in Luzon Strait. The variations in the quantities of the SIT associated with the eddy pair are examined. The main points are summarized as follows:

- Measurements showed that phase speed of the mode-1 SIT increased by up to $0.7 \mathrm{~m} \mathrm{~s}^{-1}$ inside the southern portion of the $\mathrm{AE}$, where both the eddy-induced current and stratification anomalies sped it up. On the contrary, phase speed of the mode- 1 SIT decreased by up to $0.4 \mathrm{~m} \mathrm{~s}^{-1}$ within the southern portion 


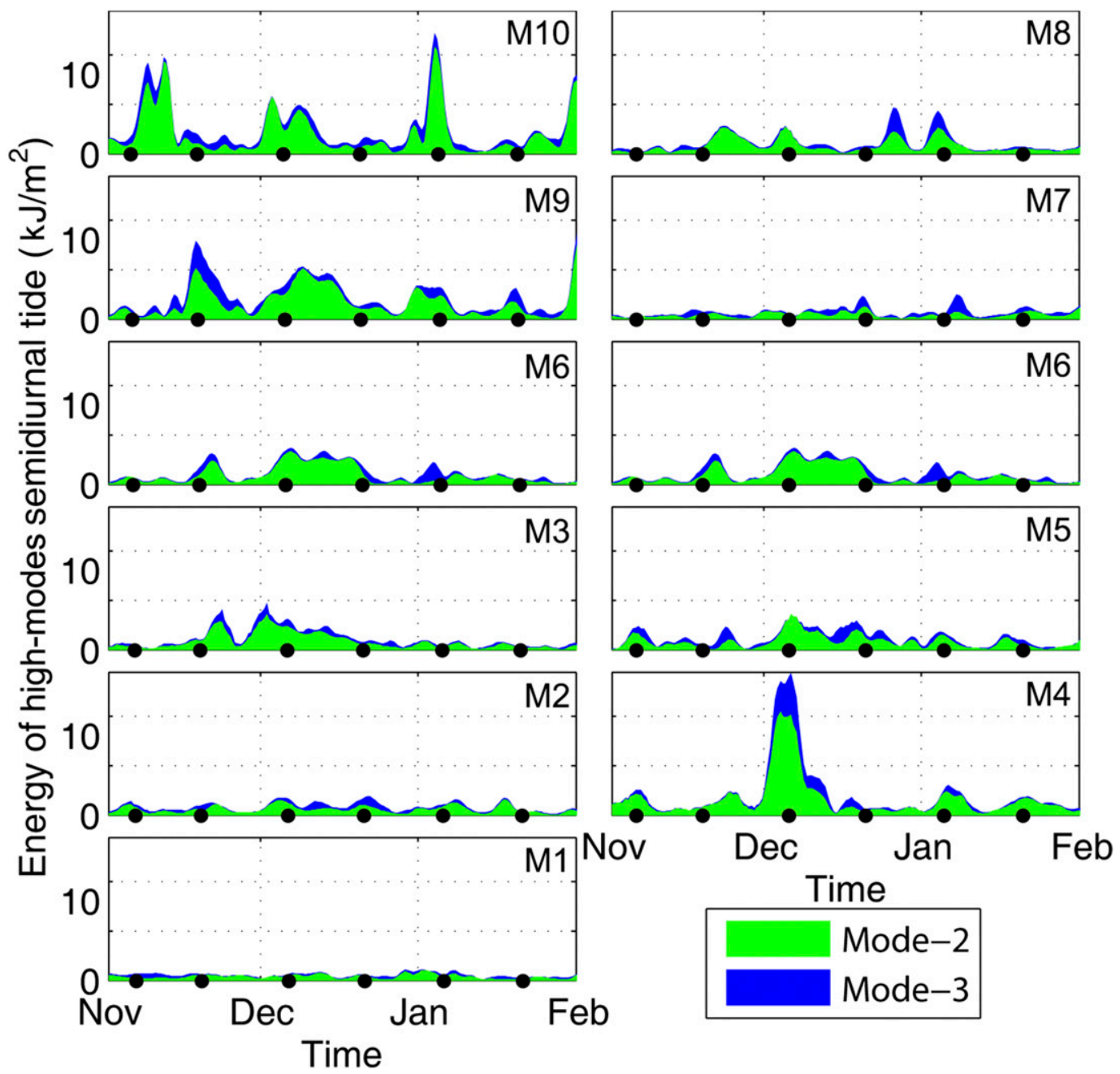

FIG. 15. (left) The depth-integrated total energy of the mode-2 and mode-3 SITs at the line of moorings in the along-beam direction. The black dots denote the spring tide periods S1-S6. (right) As in the left panels, but at the line of moorings in the cross-beam direction.

of the CE, where both the eddy-induced current and stratification anomalies slowed it down. Moreover, accompanied by the eddy current in the (reverse) direction of the SIT propagation, the mode-1 SIT propagated faster (slower) within the AE's (CE's) southern portion than within its northern portion.

- The mode-1 SIT crest exhibited different patterns due to the moving eddy pair. Generally, because the mode-1 SIT propagated faster (slower) within the AE's (CE's) southern portion than within its northern portion, the mode- 1 SIT wave crest inside the AE (CE) core were clockwise rotated (counterclockwise rotated). Moreover, on the southern (northern) boundary of the AE (CE), the wave crest exhibited a convex pattern because the mode- 1 SIT in the maximum westward-eddy-current region propagated faster than its two sides. The reverse was true on the northern (southern) boundary of the AE (CE), where the SIT wave crest showed a concave pattern.

- The depth-integrated energy flux vectors at the mooring array showed that the propagation path of the mode-1 SIT energy was highly variable in time owing to the migrating eddy pair. In mid-to-late November during the period S2, most of the mode-1 SIT energy was refracted toward the north part of the northern SCS by the AE, while in early January during the period S5, most of the mode-1 SIT energy was refracted toward the south part of the northern SCS by the CE. During these two periods, a "desert" region with the weak mode-1 SIT energy was formed around Dongsha Island. The manners in which the SIT energy propagated inside the eddies reported here can improve our understanding of the horizontal distribution and dissipation of ITs during the eddy period. 
- The observed decrease and increase in the depthintegrated mode-1 SIT energy associated with the eddies were up to $74 \%$ and $119 \%$, respectively. Owing to the energy refraction by the eddies, the depthintegrated mode-1 SIT energy generally showed concurrent responses to the eddies at the alongbeam-direction moorings but exhibited out-of-phase responses to the eddies in the south and north parts of the northern SCS at the cross-beam-direction moorings. Besides the energy refraction, the distortion of the SIT wave crest associated with the eddies also resulted in the changes in the magnitude of mode- 1 SIT energy observed at the moorings, by up to $27 \%$.

- In mid-to-late November during the period S2, the mode-1 SIT energy was refracted toward the north part of the northern SCS by the AE, which resulted in the formation of relatively large-amplitude type-B ISWs there. The amplitude of type-B ISWs in the north and south parts of the northern SCS generally varied out of phase in November and December because of the spatial variations in the SIT energy modulated by the eddies.

One important goal of S-MEE was to investigate the interactions between the internal waves and mesoscale eddies. In this study, we have shown that the mode-2 SIT in the northern SCS was intensified from late November to early January, whose energy was possibly transferred from the mode-1 SIT energy through eddy-wave interactions. However, the SIT energy flux field was not only highly temporally variable because of the moving eddies but also multidirectional because of the energy refraction by the eddies. As a result, it is difficult to quantify the energy exchanges between ITs and mesoscale eddies. In this study, we have examined the variations in the properties of the SIT associated with the eddies in both the along-beam and cross-beam directions, which is fundamental for us to investigate the energy changes between the mesoscale eddies and ITs. The energy changes between ITs and mesoscale eddies in the northern SCS will be further explored in future studies.

Acknowledgments. We thank the two anonymous reviewers for their insightful comments. The altimeter data used here are obtained from the AVISO website (http://www.aviso.oceanobs.com/duacs). The processed mooring data used in the work are available from the corresponding author on reasonable request. This study was supported by National Key Basic Research Program of China (Program 973; Grant 2014CB745003), National Key Research and Development Program (Grant 2016YFC1402605), National Natural Science Foundation of China (Grants 41506011, 41676011, and 41706005), Qingdao National Laboratory for Marine Science and Technology (Grant 2015ASKJ01), Key Research and Development Program of Shandong (Grant 2016CYJS02A03), Foundation for Innovative Research Groups of the National Natural Science Foundation of China (Grant 41521091), NSFC-Shandong Joint Fund for Marine Science Research Centers (Grant U1406402), Global Change and Air-Sea Interaction Project (Grants GASI-IPOVAI-01-03 and GASI-IPOVAI-01-02), and National Key Research and Development Program (Grant 2017YFA0603201).

\section{APPENDIX A}

\section{The Taylor-Goldstein Equation in the Presence of Background Shear and Rotation}

In the presence of background shear and rotation, Frankignoul (1970) derived the internal wave equation when the fluid was assumed to be inviscid and the Boussinesq approximation was made. Assuming that the background current is in the zonal direction, the internal wave equation [Eq. (8) in Frankignoul 1970] becomes

$$
\begin{aligned}
& D^{3}\left(\nabla^{2} w\right)-D^{2}\left(U^{\prime \prime} \frac{\partial}{\partial x} w\right)+N^{2} D\left(\nabla_{h}^{2} w\right)+D(\boldsymbol{\Omega} \nabla)^{2} w \\
& -\Omega_{3}\left(U^{\prime} \nabla\right)(\boldsymbol{\Omega} \nabla) w=0 .
\end{aligned}
$$

Here, $U(z)$ is the background current, $w$ is the vertical velocity of the internal wave, $D$ is equal to $\partial / \partial t+U \partial / \partial x$, $\nabla^{2}$ denotes the Laplacian, and $\boldsymbol{\Omega}=\left(0, \Omega_{2}, \Omega_{3}\right)$ is the rotation vector. By assuming that $w(x, z, t)=$ $\Phi(z) \exp [i(k x-\omega t)]$ and neglecting the horizontal component of the rotation vector $\Omega_{2}$ in Eq. (A1), we obtain

$$
\begin{aligned}
& \left(\omega^{3}+3 \omega k^{2} U^{2}-3 k \omega^{2} U-k^{3} U^{3}-f^{2} \omega+f^{2} k U\right) \Phi^{\prime \prime}-f^{2} k U^{\prime} \Phi^{\prime}+\left(-k^{2} \omega^{3}-3 k^{4} \omega U^{2}+3 k^{3} \omega^{2} U+k^{5} U^{3}+k \omega^{2} U^{\prime \prime}\right. \\
& \left.\quad-2 k^{2} \omega U U^{\prime \prime}+k^{3} U^{2} U^{\prime \prime}+k^{2} \omega N^{2}-N^{2} k^{3} U\right) \Phi=0,
\end{aligned}
$$

where the prime indicates the $z$ derivative and $f=\Omega_{3}$. Because $\omega=c_{p} k$, the Taylor-Goldstein equation in the presence of background shear and rotation is obtained: 


$$
\begin{aligned}
& {\left[f^{2}\left(U-c_{p}\right)-k^{2}\left(U-c_{p}\right)^{3}\right] \Phi^{\prime \prime}-f^{2} U^{\prime} \Phi^{\prime}} \\
& \quad+\left[k^{4}\left(U-c_{p}\right)^{3}+k^{2} U^{\prime \prime}\left(U-c_{p}\right)^{2}\right. \\
& \left.\quad-k^{2} N^{2}\left(U-c_{p}\right)\right] \Phi=0 .
\end{aligned}
$$

Replacing the horizontal wavenumber $k$ with $\omega / c_{p}$ and rearranging, Eq. (A3) becomes

$$
\left(B c_{p}^{4}+C c_{p}^{3}+D c_{p}^{2}+E c_{p}+F\right) \Phi=A c_{p}^{5} \Phi .
$$

Here,

$$
\begin{aligned}
& A=\omega^{2} \frac{d^{2}}{d z^{2}}-f^{2} \frac{d^{2}}{d z^{2}} \\
& B=3 \omega^{2} U \frac{d^{2}}{d z^{2}}-f^{2} U \frac{d^{2}}{d z^{2}}+f^{2} U^{\prime} \frac{d}{d z}-\omega^{2} U^{\prime \prime} \\
& C=-3 \omega^{2} U^{2} \frac{d^{2}}{d z^{2}}+\left(\omega^{4}+2 \omega^{2} U U^{\prime \prime}-\omega^{2} N^{2}\right) \\
& D=\omega^{2} U^{3} \frac{d^{2}}{d z^{2}}+\left(\omega^{2} N^{2} U-3 \omega^{4} U-\omega^{2} U^{2} U^{\prime \prime}\right) \\
& E=3 \omega^{4} U^{2}, \quad \text { and } \\
& F=-\omega^{4} U^{3}
\end{aligned}
$$

Assuming that

$$
\begin{aligned}
& \Phi_{1}=c_{p} \Phi, \\
& \Phi_{2}=c_{p} \Phi_{1}=c_{p}^{2} \Phi, \\
& \Phi_{3}=c_{p} \Phi_{2}=c_{p}^{3} \Phi, \quad \text { and } \\
& \Phi_{4}=c_{p} \Phi_{3}=c_{p}^{4} \Phi,
\end{aligned}
$$

Eq. (A4) becomes

$$
\begin{gathered}
A^{-1} B \Phi_{4}+A^{-1} C \Phi_{3}+A^{-1} D \Phi_{2} \\
+A^{-1} E \Phi_{1}+A^{-1} F \Phi=c_{p} \Phi_{4} .
\end{gathered}
$$

Equations (A6) and (A7) can be solved in the following matrix form:

$$
\begin{gathered}
\left(\begin{array}{ccccc}
0 & I & 0 & 0 & 0 \\
0 & 0 & I & 0 & 0 \\
0 & 0 & 0 & I & 0 \\
0 & 0 & 0 & 0 & I \\
A^{-1} F & A^{-1} E & A^{-1} D & A^{-1} C & A^{-1} B
\end{array}\right)\left(\begin{array}{c}
\Phi \\
\Phi_{1} \\
\Phi_{2} \\
\Phi_{3} \\
\Phi_{4}
\end{array}\right) \\
=c_{p}\left(\begin{array}{c}
\Phi \\
\Phi_{1} \\
\Phi_{2} \\
\Phi_{3} \\
\Phi_{4}
\end{array}\right) .
\end{gathered}
$$

The phase speed $c_{p}$ and normal mode of displacement $\Phi$ are derived from the real part of the computed eigenvalues and eigenvectors, respectively.

\section{APPENDIX B}

\section{Errors in the Energy and Flux Calculations}

In this study, the upper $50 \mathrm{~m}$ in the ADCP measurements are discarded because of the contamination by the acoustic reflection at the surface. On the other hand, there exists a $\sim 70$-m gap near the surface in the temperature measurement at most moorings, and at M10, there exists a $160-\mathrm{m}$ temperature measurement gap in the top. The measurement gaps near the surface may have serious impact on the precision of HKE, APE, and energy flux estimates (Nash et al. 2005). Here, we use the full-depth profiles of velocity, temperature, salinity, and pressure from the Internal Wave and Mixing Experiment (IWME) (Tian et al. 2009) to evaluate the errors in the energy and flux calculations associated with the mooring measurement gaps.

The instruments used in the IWME included a 300-kHz lowered ADCP (LADCP) and an SBE 911 plus CTD. Around 8 July 2007, a total of nine LADCP/CTD profiles were collected at a 3-h interval between successive profiles in the northern SCS at $120.50^{\circ} \mathrm{E}, 20.25^{\circ} \mathrm{N}$. As a reference, the semidiurnal energy and flux are computed from the full-depth LADCP/CTD profiles using the methods described in section $2 \mathrm{e}$ but by solving for 10 modes. At the same time, the semidiurnal energy and flux are computed by solving the first three modes for the typical case in the mooring measurements (e.g., at M3) by assuming that there exists a $50-\mathrm{m}$ gap in the velocity measurement and a 70-m gap in the temperature measurement near the surface. As shown in Fig. B1, the computed semidiurnal HKE, APE, and flux for the M3 case (center panels) exhibit similar patterns to the results using the full-depth profiles (left panels). The relative errors in the cycle mean depth-integrated mode-1 semidiurnal HKE, APE, and flux are $13 \%, 11 \%$, and $12 \%$, respectively. For the mode-2 SIT, the HKE computation at M3 has a relative error of $28 \%$.

At M10, the velocity measurement is similar to that at M3, but the temperature measurement gap near the surface is larger. In such situations, full-depth profiles of velocity and displacement at M10 are computed by solving for the first three modes and two modes, respectively. Comparing to the reference indicates that, the relative error in the computation of semidiurnal KE at M10 (right panels in Fig. B1) is $13 \%$ and 29\%, 

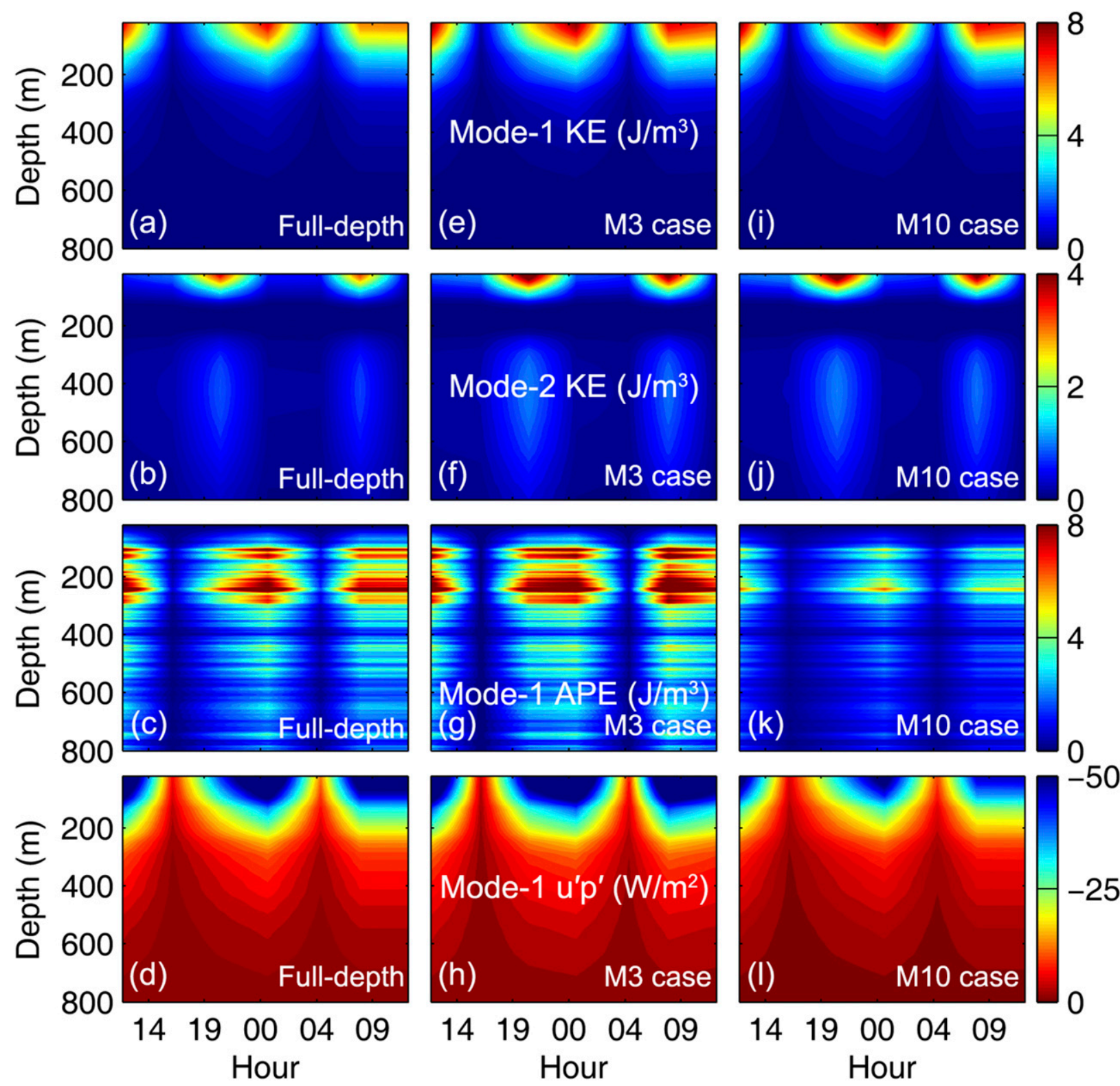

FIG. B1. (a) Mode-1 SIT HKE, (b) mode-2 SIT HKE, (c) mode-1 SIT APE, and (d) mode-1 SIT energy flux computed from the fulldepth LADCP/CTP profiles. (e)-(h) As in (a)-(d), but computed assuming that there exists a 50-m velocity measurement gap and a 70-m temperature measurement gap near the surface. (i)-(l) As in (a)-(d), but computed by assuming there exists a 50-m velocity measurement gap and a 70-m temperature measurement gap near the surface. (right) As in the left panels, but computed by assuming that there exists a 50-m velocity measurement gap and a 160-m temperature measurement gap near the surface.

respectively, for the mode- 1 and mode-2 SIT. For the mode- 1 APE and energy flux, the relative computation errors at M10 are $46 \%$ and $22 \%$, respectively. It can be seen that the error in the APE computation at M10 is relatively large.

At M5 where the ADCP data were unable to resolve the IT signals and at M6 where there is a lack of temperature measurement, the total SIT energy is obtained via $E=\left(1+r_{E}\right) \mathrm{APE}$ and $E=\left(1+1 / r_{E}\right) \mathrm{HKE}$, respectively. Using the velocity and temperature measurements at mooring M2, the errors in the energy computation at M5 and M6 are assessed. As shown in Fig. B2, the total SIT energy estimated from the HKE or APE exhibits similar changing tendencies to the observation results. On average, the relative error in the magnitude of total SIT energy estimated from HKE and APE is $20 \%$ and $15 \%$, respectively. 


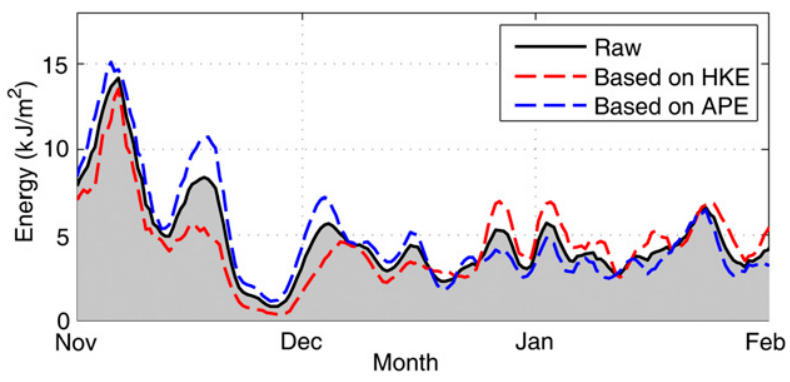

FIG. B2. Observed depth-integrated SIT energy at M2 (black). The red and blue dashed curves denote the estimated depthintegrated SIT energy based on HKE and APE, respectively.

\section{REFERENCES}

Alford, M. H., 2003: Redistribution of energy available for ocean mixing by long-range propagation of internal waves. Nature, 423, 159-162, https://doi.org/10.1038/nature01628.

— R.-C. Lien, H. Simmons, J. Klymak, S. Ramp, Y. J. Yang, D. Tang, and M.-H. Chang, 2010: Speed and evolution of nonlinear internal waves transiting the South China Sea. J. Phys. Oceanogr., 40, 1338-1355, https://doi.org/10.1175/ 2010JPO4388.1.

— Strait: Two tales of two ridges. J. Phys. Oceanogr., 41, 2211-2222, https://doi.org/10.1175/JPO-D-11-073.1.

_- and Coauthors, 2015: The formation and fate of internal waves in the South China Sea. Nature, 521, 65-69, https:// doi.org/10.1038/nature14399.

Buijsman, M. C., J. C. McWilliams, and C. R. Jackson, 2010: Eastwest asymmetry in nonlinear internal waves from Luzon Strait. J. Geophys. Res., 115, C10057, https://doi.org/10.1029/ 2009JC006004.

Chavanne, C., P. Flament, D. Luther, and K. W. Gurgel, 2010: The surface expression of semidiurnal internal tides near a strong source at Hawaii. Part II: Interactions with mesoscale currents. J. Phys. Oceanogr., 40, 1180-1200, https://doi.org/ 10.1175/2010JPO4223.1.

Chen, G., Y. Hou, and X. Chu, 2011: Mesoscale eddies in the South China Sea: Mean properties, spatiotemporal variability, and impact on thermohaline structure. J. Geophys. Res., 116, C06018, https://doi.org/10.1029/2011JD016244.

Dunphy, M., and K. G. Lamb, 2014: Focusing and vertical mode scattering of the first mode internal tide by mesoscale eddy interaction. J. Geophys. Res. Oceans, 119, 523-536, https:// doi.org/10.1002/2013JC009293.

Egbert, G. D., and R. D. Ray, 2000: Significant dissipation of tidal energy in the deep ocean inferred from satellite altimeter data. Nature, 405, 775-778, https://doi.org/10.1038/35015531.

_- , and S. Y. Erofeeva, 2002: Efficient inverse modeling of barotropic ocean tides. J. Atmos. Oceanic Technol., 19, 183-204, https://doi.org/10.1175/1520-0426(2002)019<0183: EIMOBO $>2.0 . \mathrm{CO} ; 2$.

Farmer, D., Q. Li, and J. H. Park, 2009: Internal wave observations in the South China Sea: The role of rotation and nonlinearity. Atmos.-Ocean, 47, 267-280, https://doi.org/10.3137/ OC313.2009.

Frankignoul, C. J., 1970: The effect of weak shear and rotation on internal waves. Tellus, 22, 194-204, https://doi.org/10.3402/ tellusa.v22i2.10214.
Helfrich, K. R., and R. H. J. Grimshaw, 2008: Nonlinear disintegration of the internal tide. J. Phys. Oceanogr., 38, 686-701, https://doi.org/10.1175/2007JPO3826.1.

Huang, X., W. Zhao, J. Tian, and Q. Yang, 2014: Mooring observations of internal solitary waves in the deep basin west of Luzon Strait. Acta Oceanol. Sin., 33, 82-89, https://doi.org/ 10.1007/s13131-014-0416-7.

_ Z Z. Chen, W. Zhao, Z. Zhang, C. Zhou, Q. Yang, and J. Tian, 2016: An extreme internal solitary wave event observed in the northern South China Sea. Sci. Rep., 6, 30041, https://doi.org/ 10.1038/srep30041.

—, Z. Zhang, X. Zhang, H. Qian, W. Zhao, and J. Tian, 2017: Impacts of a mesoscale eddy pair on internal solitary waves in the northern South China Sea revealed by mooring array observations. J. Phys. Oceanogr., 47, 1539-1554, https://doi.org/ 10.1175/JPO-D-16-0111.1.

Jan, S., R.-C. Lien, and C.-H. Ting, 2008: Numerical study of baroclinic tides in Luzon Strait. J. Oceanogr., 64, 789-802, https:// doi.org/10.1007/s10872-008-0066-5.

Kerry, C. G., B. S. Powell, and G. S. Carter, 2014: The impact of subtidal circulation on internal tide generation and propagation in the Philippine Sea. J. Phys. Oceanogr., 44, 1386-1405, https://doi.org/10.1175/JPO-D-13-0142.1.

Klymak, J. M., R. Pinkel, C.-T. Liu, A. K. Liu, and L. David, 2006: Prototypical solitons in the South China Sea. Geophys. Res. Lett., 33, L11607, https://doi.org/10.1029/2006GL025932.

, M. H. Alford, R. Pinkel, R.-C. Lien, Y. J. Yang, and T.-Y. Tang, 2011: The breaking and scattering of the internal tide on a continental slope. J. Phys. Oceanogr., 41, 926-945, https:// doi.org/10.1175/2010JPO4500.1.

Lelong, M. P., and J. J. Riley, 1991: Internal wave-vortical mode interactions in strongly stratified flows. J. Fluid Mech., 232, 1-19, https://doi.org/10.1017/S0022112091003609.

Li, Q., and D. M. Farmer, 2011: The generation and evolution of nonlinear internal waves in the deep basin of the South China Sea. J. Phys. Oceanogr., 41, 1345-1363, https://doi.org/10.1175/ 2011JPO4587.1.

Lien, R. C., B. Ma, Y. H. Cheng, C. R. Ho, B. Qiu, C. M. Lee, and M. H. Chang, 2014: Modulation of Kuroshio transport by mesoscale eddies at the Luzon Strait entrance. J. Geophys. Res. Oceans, 119, 2129-2142, https://doi.org/10.1002/2013JC009548.

Martini, K. I., M. H. Alford, J. D. Nash, E. Kunze, and M. A. Merrifield, 2007: Diagnosing a partly standing internal wave in Mamala Bay, Oahu. Geophys. Res. Lett., 34, L17604, https:// doi.org/10.1029/2007GL029749.

Munk, W., and C. Wunsch, 1998: Abyssal recipes II: Energetics of tidal and wind mixing. Deep-Sea Res. I, 45, 1977-2010, https:// doi.org/10.1016/S0967-0637(98)00070-3.

Nash, J. D., M. H. Alford, and E. Kunze, 2005: Estimating internal wave energy fluxes in the ocean. J. Atmos. Oceanic Technol., 22, 1551-1570, https://doi.org/10.1175/JTECH1784.1.

_ E. L. Shroyer, S. M. Kelly, and M. E. Inall, 2012: Are any coastal internal tides predictable? Oceanography, 25 (2), 80-95, https://doi.org/10.5670/oceanog.2012.44.

Park, J.-H., and D. R. Watts, 2006: Internal tides in the southwestern Japan/East Sea. J. Phys. Oceanogr., 36, 22-34, https:// doi.org/10.1175/JPO2846.1.

Pawlowicz, R., B. Beardsley, and S. Lentz, 2002: Classical tidal harmonic analysis including error estimates in MATLAB using T_TIDE. Comput. Geosci., 28, 929-937, https://doi.org/ 10.1016/S0098-3004(02)00013-4.

Ponte, A. L., and P. Klein, 2015: Incoherent signature of internal tides on sea level in idealized numerical simulations. 
Geophys. Res. Lett., 42, 1520-1526, https://doi.org/10.1002/ 2014GL062583.

Rainville, L., and R. Pinkel, 2006: Propagation of low-mode internal waves through the ocean. J. Phys. Oceanogr., 36, 1220-1236, https://doi.org/10.1175/JPO2889.1.

Ramp, S. R., Y. J. Yang, and F. L. Bahr, 2010: Characterizing the nonlinear internal wave climate in the northeastern South China Sea. Nonlinear Processes Geophys., 17, 481-498, https:// doi.org/10.5194/npg-17-481-2010.

St. Laurent, L., H. Simmons, T. Y. Tang, and Y. Wang, 2011: Turbulent properties of internal waves in the South China Sea. Oceanography, 24 (4), 78-87, https://doi.org/10.5670/ oceanog.2011.96.

Tian, J., L. Zhou, X. Zhang, X. Liang, Q. Zheng, and W. Zhao, 2003: Estimates of $M_{2}$ internal tide energy fluxes along the margin of northwestern Pacific using TOPEX/POSEIDON altimeter data. Geophys. Res. Lett., 30, 1889, https://doi.org/ 10.1029/2003GL018008.

-, Q. Yang, and W. Zhao, 2009: Enhanced diapycnal mixing in the South China Sea. J. Phys. Oceanogr., 39, 3191-3203, https://doi.org/10.1175/2009JPO3899.1.

Wang, G., J. Su, and P. C. Chu, 2003: Mesoscale eddies in the South China Sea observed with altimeter data. Geophys. Res. Lett., 30, 2121, https://doi.org/10.1029/2003GL018532.

Wang, Y.-H., C.-F. Dai, and Y.-Y. Chen, 2007: Physical and ecological processes of internal waves on an isolated reef ecosystem in the South China Sea. Geophys. Res. Lett., 34, L18609, https://doi.org/10.1029/2007GL030658.

Wu, C.-R., and T.-L. Chiang, 2007: Mesoscale eddies in the northern South China Sea. Deep-Sea Res. II, 54, 1575-1588, https://doi.org/10.1016/j.dsr2.2007.05.008.

Wunsch, C., and R. Ferrari, 2004: Vertical mixing, energy, and the general circulation of the oceans. Annu. Rev. Fluid Mech., 36, 281-314, https://doi.org/10.1146/annurev. fluid.36.050802.122121.

Yang, Q., W. Zhao, X. Liang, and J. Tian, 2016: Three-dimensional distribution of turbulent mixing in the South China Sea. J. Phys. Oceanogr., 46, 769-788, https://doi.org/10.1175/ JPO-D-14-0220.1.
Zhang, Z., W. Zhao, J. Tian, and X. Liang, 2013: A mesoscale eddy pair southwest of Taiwan and its influence on deep circulation. J. Geophys. Res. Oceans, 118, 6479-6494, https://doi.org/ 10.1002/2013JC008994.

—, J. Tian, B. Qiu, W. Zhao, P. Chang, D. Wu, and X. Wan, 2016: Observed 3D structure, generation, and dissipation of oceanic mesoscale eddies in the South China Sea. Sci. Rep., 6, 24349 , https://doi.org/10.1038/srep24349.

—, W. Zhao, B. Qiu, and J. Tian, 2017: Anticyclonic eddy sheddings from Kuroshio loop and the accompanying cyclonic eddy in the northeastern South China Sea. J. Phys. Oceanogr., 47, 1243-1259, https://doi.org/10.1175/JPO-D-16-0185.1.

Zhao, W., C. Zhou, J. Tian, Q. Yang, B. Wang, L. Xie, and T. Qu, 2014: Deep water circulation in the Luzon Strait. J. Geophys. Res. Oceans, 119, 790-804, https://doi.org/10.1002/2013JC009587.

Zhao, Z., 2014: Internal tide radiation from the Luzon Strait. J. Geophys. Res. Oceans, 119, 5434-5448, https://doi.org/ 10.1002/2014JC010014.

— - V. Klemas, Q. Zheng, and X. H. Yan, 2004: Remote sensing evidence for baroclinic tide origin of internal solitary waves in the northeastern South China Sea. Geophys. Res. Lett., 31, L22602, https://doi.org/10.1029/2004GL021061.

— M. H. Alford, J. A. MacKinnon, and R. Pinkel, 2010: Longrange propagation of the semidiurnal internal tide from the Hawaiian Ridge. J. Phys. Oceanogr., 40, 713-736, https:// doi.org/10.1175/2009JPO4207.1.

, R.-C. Lien, M. C. Gregg, and G. S. Carter, 2012: Internal tides and mixing in a submarine canyon with timevarying stratification. J. Phys. Oceanogr., 42, 2121-2142, https://doi.org/10.1175/JPO-D-12-045.1.

$\longrightarrow,-$ J. B. Girton, L. Rainville, and H. L. Simmons, 2016: Global observations of open-ocean mode- $1 \mathrm{M}_{2}$ internal tides. J. Phys. Oceanogr., 46, 1657-1684, https://doi.org/10.1175/ JPO-D-15-0105.1.

Zheng, Q., R. D. Susanto, C.-R. Ho, Y. T. Song, and Q. Xu, 2007: Statistical and dynamical analyses of generation mechanisms of solitary internal waves in the northern South China Sea. J. Geophys. Res., 112, C03021, https://doi.org/10.1029/ 2006JC003551. 